\title{
Cosmological Lepton Asymmetry, Primordial Nucleosynthesis and Sterile Neutrinos
}

\author{
Kevork Abazajian ${ }^{1,2,3}$, Nicole F. Bell ${ }^{2,3,4}$, George M. Fuller ${ }^{3,5}$, Yvonne Y. Y. Wong ${ }^{3,6}$ \\ ${ }^{1}$ Theoretical Division, MS B285, Los Alamos National Laboratory, Los Alamos NM 87545 \\ ${ }^{2}$ Theoretical Astrophysics, Fermi National Accelerator Laboratory, Batavia IL 60510 \\ ${ }^{3}$ Kavli Institute for Theoretical Physics, University of California, Santa Barbara, CA 93106 \\ ${ }^{4}$ Kellogg Radiation Laboratory, California Institute of Technology, Pasadena, CA 91125 \\ ${ }^{5}$ Department of Physics, University of California, San Diego, La Jolla, CA 92093-0319 \\ 6 Deutsches Elektronen-Synchrotron DESY, D-22607 Hamburg, Germany
}

(Dated: August 19, 2005)

\begin{abstract}
We study post weak decoupling coherent active-sterile and active-active matter-enhanced neutrino flavor transformation in the early universe. We show that flavor conversion efficiency at MikheyevSmirnov-Wolfenstein resonances is likely to be high (adiabatic evolution) for relevant neutrino parameters and energies. However, we point out that these resonances cannot sweep smoothly and continuously with the expansion of the universe. We show how neutrino flavor conversion in this way can leave both the active and sterile neutrinos with non-thermal energy spectra, and how, in turn, these distorted energy spectra can affect the neutron-to-proton ratio, primordial nucleosynthesis, and cosmological mass/closure constraints on sterile neutrinos. We demonstrate that the existence of a light sterile neutrino which mixes with active neutrinos can change fundamentally the relationship between the cosmological lepton numbers and the primordial nucleosynthesis ${ }^{4}$ He yield.
\end{abstract}

PACS numbers: 14.60.Pq; 14.60.St; 26.35.+c; 95.30.-k

\section{INTRODUCTION}

If light sterile neutrinos exist we will be forced to re-think the role of the weak interaction in the early universe, primordial nucleosynthesis, and cosmology. Though light sterile neutrinos which mix with active neutrinos long have been a subject of theoretical speculation, the LSND and KARMEN experiments 1] gave rise to particular interest in the mass-squared difference range $0.2 \mathrm{eV}^{2}<\delta m_{\text {as }}^{2}<100 \mathrm{eV}^{2}$. Here we study for this range of $\delta m_{\text {as }}^{2}$ the cosmological lepton number-driven conversion of active neutrinos, $\nu_{\alpha}$ (and/or $\left.\bar{\nu}_{\alpha}\right)$ with $\alpha=\mathrm{e}, \mu, \tau$, to a singlet, "sterile" neutrino species $\nu_{s}$ (or $\left.\bar{\nu}_{s}\right)$ in the epoch of the early universe after decoupling of the weak interactions, when neutrino spectral distortions are likely to persist.

A positive signal in the on-going mini-BooNE experiment [2], i.e., confirming the interpretation of the LSND result in terms of vacuum neutrino mixing, sets up an immediate crisis in neutrino physics. Such a result, when combined with the already well established evidence for neutrino mixing at mass-squared differences associated with the atmospheric $\left(\delta m^{2} \sim 3 \times 10^{-3} \mathrm{eV}^{2}\right)$ and solar neutrino $\left(\delta m^{2} \sim 7 \times 10^{-5} \mathrm{eV}^{2}\right)$ anomalies, would suggest the existence of three independent neutrino mass-squared differences which would, in turn, require four neutrino species. Given the $Z^{0}$-width limit on the number of flavors of neutrinos with standard weak interactions (3), a fourth neutrino would have to be "sterile," with subweak interaction strength, e.g., perhaps an $\mathrm{SU}(2)$ singlet. The only alternative to this line of reasoning and to this conclusion is the possibility of CPT violation 3]. However, there is no consistency of the neutrino oscillation data with a CPT-violating three-neutrino model at a $3-\sigma$ level [4].

Hand in hand with this particle physics dilemma, evidence for a singlet neutrino that mixes with active neutrinos in this mass-squared range also confronts cosmology with a curious and vexing problem. In the standard cosmological model with zero or near-zero net lepton numbers one would expect that matter-supressed neutrino oscillations in the channel $\nu_{\alpha} \rightleftharpoons \nu_{s}$ or in $\bar{\nu}_{\alpha} \rightleftharpoons \bar{\nu}_{s}$ (where $\alpha=e, \mu, \tau)$ proceeding in the regime above weak interaction decoupling $(T>3 \mathrm{MeV})$, would efficiently populate seas of singlet neutrinos [5]. The significant additional energy density in these sterile neutrino seas would engender a faster expansion rate for the universe and a consequently higher temperature for Weak Freeze-Out (where the initial isospin of the universe, the neutron-to-proton ratio is set). A higher Weak Freeze-Out temperature would result in more neutrons and, hence, a higher yield of ${ }^{4} \mathrm{He}$.

A higher predicted abundance of ${ }^{4} \mathrm{He}$ arguably may be in conflict (or is close to being in conflict) with the observationally inferred upper limit on the primordial helium abundance. Depending on the helium abundance inferred from compact blue galaxies, an increase in the predicted Big Bang Nucleosynthesis (BBN) ${ }^{4} \mathrm{He}$ yield may or may not be disfavored [5, 6]. However, the primordial helium abundance is notoriously difficult to extract from the observational data and recent studies point to a fair range for the observationally inferred primordial mass helium 
fraction: $23 \%$ to $26 \%$ [7]. The upper limit of this range is provocatively close to the standard $\mathrm{BBN}{ }^{4} \mathrm{He}$ mass fraction yield prediction, $24.85 \pm 0.05 \%$, as computed with the deuterium-determined or CMB (Cosmic Microwave Background) anisotropy-determined baryon density.

Additionally, it has been suggested [8] that a fully populated sea of sterile neutrinos and antineutrinos with rest masses $\sim \sqrt{\delta m_{\text {as }}^{2}}$ could be in conflict with neutrino mass bounds derived from CMB anisotropy limits and large scale structure considerations [9]. There is a recent analysis of constraints from measurements of galaxy bias stemming from galaxy-galaxy lensing and the inferred linear matter power spectrum derived from the Lyman alpha forest in the Sloan Digital Sky Survey (SDSS) 10]. This analysis specifically considers a so-called " $3+1$ " neutrino mass hierarchy, i.e., the scheme which is appropriate for constraining sterile neutrinos. The neutrino mass constraint so derived is somewhat less stringent than constraints in schemes with three neutrinos with degenerate masses. However, the central conclusions of Ref. [8] survive.

Should we someday be confronted with a positive indication of neutrino flavor mixing with mass-squared scale consistent with the range for $\delta m_{\text {as }}^{2}$, we will have a problem that would call for modification either of our notions of basic neutrino physics or of the standard cosmological model. There have been a number of ways proposed to get out of these cosmological difficulties. For instance, if neutrinos do not acquire mass until after the BBN epoch, as may occur via a late-time phase transition [11, 12, the singlet states will not be populated via oscillations during the BBN era. In addition to schemes involving the epoch of neutrino mass generation [13], annihilation 14] or decay 12] of the singlet neutrinos (when $m_{s} \sim T$ ) may alleviate or avoid the CMB and large scale structure constraints. However, chief among the mechanisms proposed to escape the cosmological difficulties associated with singlet neutrinos, is the the invocation of a significant net lepton number 15].

The idea is that the net lepton number gives active neutrinos larger effective masses in medium in the early universe, thereby driving them further off-resonance in the epoch prior to weak decoupling (i.e., $T>3 \mathrm{MeV}$ ) and reducing their effective matter mixing angles with the singlet neutrino. In turn, smaller effective matter mixing angles would imply a suppressed production of singlet neutrinos and, hence, a reduced population of the singlet neutrino sea. This lepton number-induced suppression of active-sterile mixing at high temperature is why we assume here that there is no initial population of the sterile neutrino sea.

The lepton number residing in the sea of $\nu_{\alpha}$ and $\bar{\nu}_{\alpha}$ neutrinos $(\alpha=e, \mu, \tau)$ is defined in analogy to the baryon number $\eta \equiv\left(n_{b}-n_{\bar{b}}\right) / n_{\gamma}$,

$$
L_{\nu_{\alpha}}=\frac{n_{\nu_{\alpha}}-n_{\bar{\nu}_{\alpha}}}{n_{\gamma}}
$$

where $n_{\gamma}=\left(2 \zeta(3) / \pi^{2}\right) T_{\gamma}^{3}$ is the proper photon number density at temperature $T_{\gamma}$, and where $n_{\nu_{\alpha}}$ and $n_{\bar{\nu}_{\alpha}}$ are the number densities of $\nu_{\alpha}$ and $\bar{\nu}_{\alpha}$ neutrinos, respectively, at this epoch. After the epoch of $e^{ \pm}$annihilation the baryon number is $\eta \approx 6 \times 10^{-10}$, whereas at earlier epochs it is roughly two and half times larger. We will consider here net lepton numbers which are vastly larger than $\eta$, so its precise value is of no consequence for our results.

We can insure that the effective matter mixing angles for the oscillation channel $\nu_{\alpha} \rightleftharpoons \nu_{s}$ (or $\bar{\nu}_{\alpha} \rightleftharpoons \bar{\nu}_{s}$ ) are sufficiently small to suppress singlet neutrino production if the Mikheyev-Smirnov-Wolfenstein (MSW) 16] resonance temperature is less than the weak decoupling temperature, $T_{\text {res }}<T_{\mathrm{dec}}$. This implies that the lepton number associated with any of the active neutrino flavors should satisfy,

$$
L>\frac{10^{-3}}{\epsilon}\left(\frac{2}{N_{\text {degen }}}\right)\left(\frac{3 \mathrm{MeV}}{T_{\mathrm{dec}}}\right)^{4}\left(\frac{\delta m_{\mathrm{as}}^{2} \cos 2 \theta}{1 \mathrm{eV}^{2}}\right)
$$

where $\theta$ is the vacuum mixing angle characteristic of $\nu_{\alpha} \rightleftharpoons \nu_{s}$ oscillations, $N_{\text {degen }}$ is the number of neutrino species possessing this lepton number, and where $\epsilon \equiv E_{\nu} / T$. For neutrinos with typical energies in the early universe (i.e., $\epsilon \sim 1$ ), suppression of singlet neutrino production would require lepton numbers ranging from $L>10^{-4}$ for $\delta m_{a s}^{2}=0.2 \mathrm{eV}^{2}$ to $L>5 \times 10^{-3}$ for $\delta m_{\text {as }}^{2}=10 \mathrm{eV}^{2}$. Current limits on lepton numbers are $\left|L_{\nu_{\alpha}}\right|<0.1$ [17] (and possibly even weaker by a factor of two or so if allowance is made for another source of extra energy density in the early universe 18, 19]). Therefore, this avenue for escape from the sterile neutrino conundrum appears to be allowed, albeit at the cost of a huge disparity between the lepton and baryon numbers.

However, this argument overlooks an important point. Though the large lepton number supresses the effective matter mixing angle for $\nu_{\alpha} \rightleftharpoons \nu_{s}$ during the epoch of the early universe where active neutrinos are thermally coupled $\left(T>T_{\text {dec }}\right)$, it can cause coherent matterenhancement of this channel at lower temperatures where the active neutrinos rarely scatter and are effectively decoupled. Resonant MSW transformation of active neutrinos to singlets in the channel $\nu_{\alpha} \rightleftharpoons \nu_{s}$ is, however, self limiting. This is because as the universe expands and the resonance sweeps from low toward higher neutrino energy, the conversion of $\nu_{\alpha}$ 's decreases the lepton number which, in turn, causes the resonance sweep rate to increase, eventually causing neutrinos to evolve non-adiabatically through resonance and so causing flavor transformation to cease.

At issue then is how many active neutrinos can be converted to sterile neutrinos prior to or during the epoch where the neutron-to-proton ratio is set ("Weak FreezeOut"). If there is a significant conversion, the resultant non-thermal active neutrino energy spectra can cause an increase or decrease (if $\bar{\nu}_{\alpha} \rightleftharpoons \bar{\nu}_{s}$ is enhanced) in the ${ }^{4} \mathrm{He}$ yield and call into question the viability of invoking a large net lepton number to reconcile neutrino physics and BBN. Other but related aspects of transformationinduced nonthermal neutrino spectra effects on primor- 
dial nucleosynthesis have been studied in Ref.s [20, 21]. Likewise, previous studies have considered other aspects of the relationship between sterile neutrinos and BBN 22], as well as constraints on sterile neutrinos without a primordial lepton number [23]. In any case, non-thermal energy distribution functions for $\nu_{e}$ and/or $\bar{\nu}_{e}$ change the relationship between the $\mathrm{BBN}{ }^{4} \mathrm{He}$ yield and the neutrino chemical potentials.

In section II we discuss the physics of active-sterile neutrino flavor transformation in the early universe and point out a key issue in how the MSW resonance sweeps through the neutrino energy distribution functions as the universe expands. The generally high adiabaticity of neutrino flavor evolution is also pointed out in this section. Simultaneous active-active and active-sterile neutrino flavor conversion, and "synchronization" are also discussed in this section. Possible multi-neutrino mass level crossing scenarios in the early universe are discussed in this section. Sterile neutrino contributions to closure, constraints on this from large scale structure and Cosmic Microwave Background radiation considerations, as well as other sterile neutrino sea population constraints are examined in section III. In section IV we describe how distorted $\nu_{e}$ and/or $\bar{\nu}_{e}$ distribution functions impact the rates of the lepton capture reactions that determine the neutron-to-proton ratio and the ${ }^{4} \mathrm{He}$ yield in BBN. This is then applied in various initial lepton number and neutrino conversion scenarios. Finally, in section $\mathrm{V}$ we give conclusions and speculations regarding the neutrino mass and cosmological lepton number insights that would follow in the wake of an experimental signature for a large neutrino mass-squared difference of order the range given for $\delta m_{a s}^{2}$. Appendix A provides an exposition of the lepton capture rates on free nucleons when, as appropriate, $\nu_{e}$ or $\bar{\nu}_{e}$ energy distribution functions are zero up to some energy, and thermal/Fermi-Dirac at higher energies.

\section{COHERENT NEUTRINO FLAVOR TRANSFORMATION IN THE EARLY UNIVERSE}

Coherent conversion of active neutrino species into singlets in the early universe can occur through the usual
MSW process, albeit in an exotic setting. This process can be described simply: (1) an active neutrino (mostly the light mass state in vacuum) forward scatters on particles in the plasma and, if there is a net lepton and/or baryon number, will acquire a positive effective mass; (2) an MSW resonance (mass level crossing, where in-medium mixing is large) can occur when this effective mass is close to the mass associated with the singlet (mostly the heavy mass state). This process is discussed in subsection A, but despite the simplicity of the physics behind it, the neutrino energy dependence/history of MSW resonances in the early universe can be quite complex, as shown in subsection B.

The efficiency of flavor conversion at a mass level crossing depends on the ratio of the resonance width (in time or space) to the neutrino oscillation length. Efficient, adiabatic conversion takes place only when this ratio is large, and in subsection $\mathrm{C}$ we examine this physics in detail for our particular problem.

Active-active matter-enhanced neutrino flavor conversion, discussed in subsection $\mathrm{D}$, can occur simultaneously with active-sterile transformation in the early universe. This can greatly complicate computing the history of the neutrino distribution functions. Subsection E below deals with the limit where active-active conversion is efficient, while subsection $\mathrm{F}$ examines flavor evolution in the limit where this conversion channel is inefficient.

\section{A. Neutrino Effective Masses and Level Crossings}

The forward charged and neutral current exchange Hamiltonians for the neutrinos in the early universe are as follows (see, e.g., [24]):

$$
H\left(\nu_{s}\right)=0
$$

$$
\begin{gathered}
H\left(\nu_{e}\right)=\sqrt{2} G_{\mathrm{F}}\left(n_{e}-\frac{1}{2} n_{n}\right)+\sqrt{2} G_{\mathrm{F}}\left(2\left(n_{\nu_{e}}-n_{\bar{\nu}_{e}}\right)+\left(n_{\nu_{\mu}}-n_{\bar{\nu}_{\mu}}\right)+\left(n_{\nu_{\tau}}-n_{\bar{\nu}_{\tau}}\right)\right) \\
H\left(\nu_{\mu}\right)=\sqrt{2} G_{\mathrm{F}}\left(-\frac{1}{2} n_{n}\right)+\sqrt{2} G_{\mathrm{F}}\left(\left(n_{\nu_{e}}-n_{\bar{\nu}_{e}}\right)+2\left(n_{\nu_{\mu}}-n_{\bar{\nu}_{\mu}}\right)+\left(n_{\nu_{\tau}}-n_{\bar{\nu}_{\tau}}\right)\right) \\
H\left(\nu_{\tau}\right)=\sqrt{2} G_{\mathrm{F}}\left(-\frac{1}{2} n_{n}\right)+\sqrt{2} G_{\mathrm{F}}\left(\left(n_{\nu_{e}}-n_{\bar{\nu}_{e}}\right)+\left(n_{\nu_{\mu}}-n_{\bar{\nu}_{\mu}}\right)+2\left(n_{\nu_{\tau}}-n_{\bar{\nu}_{\tau}}\right)\right) .
\end{gathered}
$$

Here $n_{e}=n_{e^{-}}-n_{e^{+}}$is the net number density of electrons, $n_{n}=n_{b}-n_{p}$ is the number density of neutrons, and $n_{b}$ and $n_{p}$ are the net number densities of baryons 
and protons, respectively. Charge neutrality implies that the number density of protons is $n_{p}=n_{e}=n_{b} Y_{e}$. The net number of electrons per baryon is $Y_{e}$. The baryon number density is $n_{b} \approx \eta n_{\gamma}$, where the baryon-to-photon ratio $\eta$ is as defined above.

Weak Decoupling occurs when neutrino scattering becomes so slow that it can no longer facilitate efficient energy exchange between the neutrino gas and the plasma. For the low lepton numbers considered here, Weak Decoupling occurs around temperature $T \sim 3 \mathrm{MeV}$, though this decoupling process takes place over a range in temperature of a few $\mathrm{MeV}$.

Weak Freeze-Out occurs when the rates of the reactions that govern the ratio of neutrons-to-protons $(n / p=$ $\left.1 / Y_{e}-1\right)$ fall below the expansion rate of the universe. This is usually taken to be $T \approx 0.7 \mathrm{MeV}$ for standard cosmological parameters. However, below this temperature $Y_{e}$ continues to be modified by lepton capture and/or free neutron decay as discussed below.

Note that for temperatures well above Weak Decoupling, we have $Y_{e} \approx 0.5$, i.e., nearly equal numbers of neutrons and protons. We could then approximate $n_{e}-\frac{1}{2} n_{n}=n_{b}\left(\frac{3}{2} Y_{e}-\frac{1}{2}\right) \approx n_{\gamma} \eta / 4$, and $-\frac{1}{2} n_{n}=n_{b}\left(Y_{e} / 2-\right.$ $\left.\frac{1}{2}\right) \approx-n_{\gamma} \eta / 4$. We will use this approximation in what follows even for the epoch below weak decoupling where it is not numerically accurate. This will result in no loss of accuracy in the full calculation because we consider large net lepton numbers, $L_{\nu_{\alpha}} \gg \eta$.

We can denote the weak potentials from neutrinoelectron charged current forward exchange scattering and neutrino-neutrino neutral current forward exchange scattering as $A$ and $B$, respectively, with their sum being

$$
A+B \approx \frac{2 \sqrt{2} \zeta(3) G_{\mathrm{F}} T^{3}}{\pi^{2}}\left(\mathcal{L} \pm \frac{\eta}{4}\right)
$$

where $G_{\mathrm{F}}$ is the Fermi constant, the Riemann Zeta function of argument 3 is $\zeta(3) \approx 1.20206$, and we take the plus sign for transformation of $\nu_{e}$, and the minus sign for conversion of $\nu_{\mu}$ and/or $\nu_{\tau}$. (Here the plus sign is taken when we intend $A+B=H\left(\nu_{e}\right)$ and the minus sign is taken when $A+B=H\left(\nu_{\mu, \tau}\right)$.) A measure of the lepton number which enters into the potential for the $\nu_{\alpha} \rightleftharpoons \nu_{s}$ $(\alpha=e, \mu, \tau)$ conversion channel is

$$
\mathcal{L} \equiv 2 L_{\nu_{\alpha}}+\sum_{\beta \neq \alpha} L_{\nu_{\beta}}
$$

We will refer to this quantity as the "potential lepton number." In general this may be different for different channels $\nu_{\alpha} \rightleftharpoons \nu_{s}$, even for a given set of lepton numbers associated with each flavor.

Finally, since the early universe is at relatively high entropy per baryon, the overall weak potential has a contribution from neutrino neutral current forward scattering on a thermal lepton background. This thermal potential is

$$
C \approx-r_{\alpha} G_{\mathrm{F}}^{2} \epsilon T^{5}
$$

where the neutrino energy divided by the temperature is $\epsilon \equiv E_{\nu} / T$. For the conversion channel $\nu_{e} \rightleftharpoons \nu_{s}$, we employ $r_{e}^{0} \approx 79.34$, while for the channel $\nu_{\mu, \tau} \rightleftharpoons \nu_{s}$, we use $r_{\mu, \tau}^{0} \approx 22.22$. If the neutrinos have strictly thermal energy distribution functions, then

$$
r_{\alpha} \approx r_{\alpha}^{0}\left[\frac{F_{2}\left(\eta_{\nu_{\alpha}}\right)}{F_{2}(0)}+\frac{F_{2}\left(\eta_{\bar{\nu}_{\alpha}}\right)}{F_{2}(0)}\right]
$$

where the neutrino and antineutrino degeneracy parameters are $\eta_{\nu_{\alpha}}$ and $\eta_{\bar{\nu}_{\alpha}}$, respectively, and the Fermi integrals of order 2 are defined below. The approximations $r_{e} \approx r_{e}^{0}$ and $r_{\mu, \tau} \approx r_{\mu, \tau}^{0}$ suffice for lepton numbers below the conventional limits.

The total weak forward scattering potential is

$$
V=A+B+C
$$

For the transformation channel $\nu_{\alpha} \rightleftharpoons \nu_{s}$, the neutrino mass level crossing (MSW resonance) condition for a neutrino with scaled energy $\epsilon$ is

$$
\frac{\delta m^{2} \cos 2 \theta}{2 \epsilon T}=V
$$

where $\delta m^{2}$ is the difference of the squares of the appropriate neutrino mass eigenvalues and $\theta$ is the relevant effective two-by-two vacuum mixing angle. Neglecting the light mass eigenvalue, the effective mass-squared acquired by an electron neutrino from forward scattering on weak charge-carrying targets in the early universe is

$$
m_{\mathrm{eff}}^{2} \approx 2 \epsilon V \approx\left(8.03 \times 10^{-12} \mathrm{MeV}^{2}\right) \epsilon(\mathcal{L} \pm \eta / 4)\left(\frac{T}{\mathrm{MeV}}\right)^{4}-\left(2.16 \times 10^{-20} \mathrm{MeV}^{2}\right) \epsilon^{2}\left(\frac{T}{\mathrm{MeV}}\right)^{6}
$$

It is clear that we can negelect the second term (the thermal term $C$ ) in Eq. (11) in the regime between Weak Decoupling and Weak Freeze-Out, where $3 \mathrm{MeV}>T>$
0.7 MeV. We also neglect the baryon/electron term, $\pm \eta / 4$. 
At a given temperature, the scaled neutrino energy which is resonant is then

$$
\epsilon_{\mathrm{res}}=\frac{\delta m^{2} \cos 2 \theta}{2 V T}
$$

The dependence of resonant neutrino energy on temperature and lepton number is

$$
\begin{aligned}
\epsilon_{\mathrm{res}} & \approx \frac{\pi^{2} \delta m^{2} \cos 2 \theta}{2^{5 / 2} \zeta(3) G_{\mathrm{F}}(\mathcal{L} \pm \eta / 4) T^{4}} \\
& \approx 0.124\left(\frac{\delta m^{2} \cos 2 \theta}{1 \mathrm{eV}^{2}}\right) \frac{1}{\mathcal{L}}\left(\frac{\mathrm{MeV}}{T}\right)^{4} .
\end{aligned}
$$

It is clear from Eq. (15) that as the universe expands and the temperature drops, the resonance energy $\epsilon_{\text {res }}$ will sweep from lower to higher values. In fact, as the resonance sweeps through the active neutrino distribution, converting $\nu_{\alpha} \rightarrow \nu_{s}, \mathcal{L}$ will decrease, further accelerating the resonance sweep rate.

Assuming homogeneity and isotropy, the number density of active neutrinos $\nu_{\alpha}$ with thermal distribution function $f_{\nu_{\alpha}}(\epsilon)$ in the scaled energy range $\epsilon$ to $\epsilon+d \epsilon$ is

$$
d n_{\nu_{\alpha}}=n_{\nu_{\alpha}} f_{\nu_{\alpha}}(\epsilon) d \epsilon
$$

where $n_{\nu_{\alpha}}$ is the total number density (that is, integrated over all neutrino energies). In terms of the temperature $T$ and degeneracy parameter $\eta_{\nu_{\alpha}} \equiv \mu_{\nu_{\alpha}} / T$, where $\mu_{\nu_{\alpha}}$ is the appropriate chemical potential, the thermal distribution function is

$$
f_{\nu_{\alpha}}(\epsilon)=\frac{1}{F_{2}\left(\eta_{\nu_{\alpha}}\right)} \frac{\epsilon^{2}}{e^{\epsilon-\eta_{\nu_{\alpha}}+1}} .
$$

We define relativistic Fermi integrals of order $k$ in the usual fashion:

$$
F_{k}(\eta) \equiv \int_{0}^{\infty} \frac{x^{k} d x}{e^{x-\eta}+1}
$$

The total number density of thermally distributed active neutrinos $\nu_{\alpha}$ with temperature $T_{\nu}$ and degeneracy parameter $\eta_{\nu_{\alpha}}$ is

$$
n_{\nu_{\alpha}}=\frac{T_{\nu}^{3}}{2 \pi^{2}} F_{2}\left(\eta_{\nu_{\alpha}}\right)
$$

Note that if the neutrino degeneracy parameter is $\eta_{\nu_{\alpha}}=$ 0 , then $F_{2}(0)=3 \zeta(3) / 2$ and the number density of thermally distributed $\nu_{\alpha}$ 's is

$$
n_{\nu_{\alpha}}=\frac{3}{8} n_{\gamma}\left(\frac{T_{\nu}}{T_{\gamma}}\right)^{3}
$$

where we allow for the neutrino temperature $T_{\nu}$ to differ from the photon/plasma temperature $T_{\gamma}$.

The relationship between the lepton number in $\alpha$ flavor neutrinos and the $\nu_{\alpha}$ degeneracy parameter is

$$
L_{\nu_{\alpha}}=\left(\frac{\pi^{2}}{12 \zeta(3)}\right)\left(\frac{T_{\nu}}{T_{\gamma}}\right)^{3}\left[\eta_{\nu_{\alpha}}+\eta_{\nu_{\alpha}}^{3} / \pi^{2}\right]
$$

This relation assumes that neutrinos have Fermi-Dirac energy spectra and that $\nu_{\alpha}$ and antineutrinos $\bar{\nu}_{\alpha}$ are (or were at one point) in thermal and chemical equilibrium so that $\eta_{\bar{\nu}_{\alpha}}=-\eta_{\nu_{\alpha}}$. In the limit where the lepton number is small, so that $\eta_{\nu_{\alpha}} \ll 1$, and the neutrino and photon temperatures are nearly the same, we can approximate Eq. (21) as $\eta_{\nu_{\alpha}} \approx 1.46 L_{\nu_{\alpha}}$. Neutrino degeracy parameter is a comoving invariant; whereas, lepton number is not in general since the photons can be heated relative to the neutrinos by, e.g., $e^{ \pm}$annihilation.

\section{B. Lepton Number Depletion and the Time/Temperature Dependence of Resonance Energies}

As the universe expands and $\nu_{\alpha}$ neutrinos are converted to sterile species $\nu_{s}$, the lepton number $L_{\nu_{\alpha}}$ drops. As $\mathcal{L}$ approaches zero, the resonance sweep rate becomes so large that neutrinos will be propagating through MSW resonances non-adiabatically [25]. Efficient neutrino flavor conversion ceases at this point. If the conversion process results in a change in the number density of $\nu_{\alpha}$ neutrinos, $\Delta n_{\nu_{\alpha}}$, such that the lepton number associated with this species changes by $\Delta L_{\nu_{\alpha}}=-\Delta n_{\nu_{\alpha}} / n_{\gamma}$, then the potential lepton number would change from its initial value, $\mathcal{L}^{\text {initial }}$ to

$$
\mathcal{L}^{\text {final }}=\mathcal{L}^{\text {initial }}+2 \Delta L_{\nu_{\alpha}} .
$$

The adiabaticity condition ensures that flavor conversion ceases when $\mathcal{L}^{\text {final }}$ approaches zero, or in other words, when $\Delta L_{\nu_{\alpha}}=-\mathcal{L}^{\text {initial }} / 2$. It is important to note that transformation of any flavor active neutrino to sterile flavor can drive down the overall potential lepton number, no matter which flavor or flavors of active neutrinos harbor the net lepton number.

If additionally we were to assume that the resonance smoothly and continuously swept through the $\nu_{\alpha}$ energy distribution from zero to scaled energy $\epsilon$ during this conversion process, we would have $\Delta n_{\nu_{\alpha}}=\int_{0}^{\epsilon} d n_{\nu_{\alpha}}$ and so the concomitant change in lepton number would be

$$
\Delta L_{\nu_{\alpha}} \approx-\frac{3}{8}\left(\frac{T_{\nu}}{T_{\gamma}}\right)^{3} \frac{1}{F_{2}(0)} \int_{0}^{\epsilon} \frac{x^{2}}{e^{x-\eta_{\nu_{\alpha}}+1}} d x .
$$

In this idealized scenario, the potential lepton number as a function of $\epsilon$ is

$$
\mathcal{L}(\epsilon) \approx \mathcal{L}^{\text {initial }}-\frac{3}{4} \frac{1}{F_{2}(0)} \int_{0}^{\epsilon} \frac{x^{2}}{e^{x-\eta_{\nu_{\alpha}}+1}} d x .
$$

In this last relation we have set the photon/plasma and neutrino temperatures to be the same. This is a good approximation in the epoch where it turns out we will be most interested in resonance sweep, between Weak Decoupling and $T \approx 0.5 \mathrm{MeV}$. During this time there has been little annihilation of $e^{ \pm}$pairs and, consequently, little heating of the photons/plasma relative to the decoupled neutrinos. 


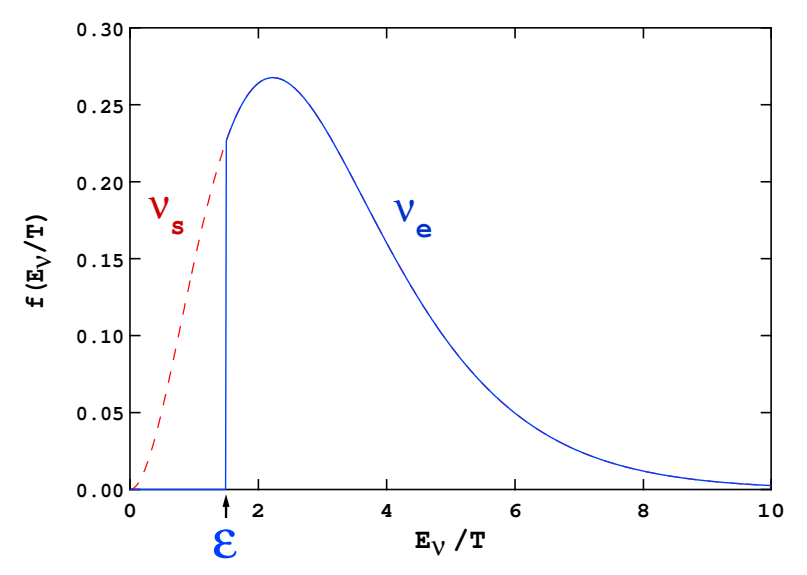

FIG. 1: The nonthermal scaled energy $\left(E_{\nu} / T\right)$ distributions $f\left(E_{\nu} / T\right)$ for $\nu_{s}$ (dashed) and $\nu_{e}$ (solid) resulting from smooth, adiabatic resonance sweep from $E_{\nu} / T=0$ to $E_{\nu} / T=\epsilon$.

Employing the approximation of a smooth and continuous sweep of scaled resonance energy from zero to $\epsilon$, we can re-write the resonance condition, Eq. (15), as

$$
\epsilon \mathcal{L}(\epsilon) \approx \frac{\pi^{2} \delta m^{2} \cos 2 \theta}{2^{5 / 2} \zeta(3) G_{\mathrm{F}} T^{4}}
$$

For smooth, continuous and adiabatic (i.e., complete conversion) resonance sweep up to a scaled energy $\epsilon$, the resulting active and sterile neutrino distribution functions would be as shown in Fig. (11). This energy distribution function is zero for all values of scaled neutrino energy $0 \leq E_{\nu} / T \leq \epsilon$, and has a normal Fermi-Dirac thermal distribution character for all neutrino energies $E_{\nu} / T>\epsilon$. The corresponding sterile neutrino energy spectrum would be the "mirror image" of this: a thermal Fermi-Dirac spectrum for $0 \leq E_{\nu} / T \leq \epsilon$, and zero occupation for $E_{\nu} / T>\epsilon$.

However, Eq. (25) reveals a problem: the resonance cannot sweep continuously and smoothly to the point where $\mathcal{L}(\epsilon) \rightarrow 0$. This is because $\epsilon \mathcal{L}(\epsilon)$ is a peaked function. The maximum of this function occurs for a value $\epsilon_{\max }$ satisfying the integral equation

$$
\epsilon_{\max }^{3} \approx 2 \zeta(3)\left(e^{\epsilon_{\max }-\eta_{\nu_{\alpha}}}+1\right) \mathcal{L}\left(\epsilon_{\max }\right) .
$$

It is clear, however, that as the universe expands, the right hand side of Eq. (25) will increase monotonically. Although the resonance sweep can begin smoothly and continuously, there will come a point where it is no longer possible to find a solution to Eq. (25). This will occur when the resonance energy reaches $\epsilon_{\max }$.

Fig. (2) shows graphically the problem of obtaining a solution to Eq. (25) for a particular case. The solid line in this figure is $\epsilon \mathcal{L}(\epsilon)$ as computed by assuming a smooth and continuous resonance sweep scenario. Here we have chosen initial lepton numbers $L_{\nu_{e}}=L_{\nu_{\mu}}=$ $L_{\nu_{\tau}}=0.01096$. This corresponds to an initial potential lepton number $\mathcal{L}(\epsilon=0)=0.04384$. The arrows in this figure give the sense of evolution along the solid curve as the universe expands and the net potential lepton number decreases as a result of neutrino flavor conversion in the channel $\nu_{e} \rightarrow \nu_{s}$. The maximum value on this curve occurs at $\epsilon_{\max } \approx 0.598$.

However, the potential lepton number would be completely depleted (i.e., $\left.\mathcal{L}\left(\epsilon_{\text {c.o. }}\right)=0\right)$ in the smooth and continuous resonance sweep scenario only when $\epsilon$ reaches a "cut-off" value $\epsilon_{\text {c.o. }}$, which is $\approx 0.987$ in this case. The horizontal dashed lines in Fig. (2) correspond to values of the right-hand side of Eq. (25) for epochs of the universe corresponding to temperatures $T=2.0 \mathrm{MeV}$, $T=1.595 \mathrm{MeV}$, and $T=1.5 \mathrm{MeV}$. Solutions to this equation are possible when these curves cross the solid $\epsilon L(\epsilon)$ curve. Clearly, no solutions are possible in the continuous resonance sweep scenario for $T<1.6 \mathrm{MeV}$ in this case.

What happens beyond this point, e.g., for $T<$ $1.6 \mathrm{MeV}$ ? If we relax the demand that the resonance sweep be continuous, then it is possible in principle to find a solution to Eq. (25) as the temperature drops beyond the point where $\epsilon=\epsilon_{\max }$, though this would require that the product of scaled resonance energy and potential lepton number differ from the solid curve $\epsilon \mathcal{L}(\epsilon)$. Though a detailed numerical model of this process is beyond the scope of this work, we can get a rough idea of what might happen with the following argument.

Suppose we take a time step resulting in a new temperature $T^{\prime}$ slightly lower than $T_{\max }$, the temperature where the last continuous sweep solution exists, i.e., where $\epsilon=\epsilon_{\max }\left(T_{\max } \approx 1.6 \mathrm{MeV}\right.$ for our example case $)$ and $\mathcal{L}=\mathcal{L}\left(\epsilon_{\max }\right)$. One possibility is that the resonance energy could skip to some value $\epsilon>\epsilon_{\max }$, toward the higher energy portion of the neutrino distribution function. In this way the product $\epsilon \mathcal{L}\left(\epsilon_{\max }\right)$ could be large enough to match the right-hand side of Eq. (25) at the new temperature $T^{\prime}$. Of course, this would result in resonant neutrino flavor conversion and so $\mathcal{L}$ would be lowered and eventually we again would be unable to maintain a smooth resonance sweep. At that point the resonance energy could skip again discontinuously, etc.. It is possible that beyond $\epsilon_{\max }$ the resonance sweeps stochastically in this way through relatively small intervals of the active neutrino distribution function leaving a "picket fence" distribution beyond $\epsilon_{\max }$.

Though the details may differ from this simple scheme, we believe that resonant neutrino flavor conversion for $\epsilon>\epsilon_{\max }$ will occur because: (1) as we show below, neutrino flavor evolution at this epoch is very adiabatic for relevant neutrino parameters; and (2) the resonance condition can be met for some value of neutrino energy so long as the net lepton number is non-zero. In any case, however, active-to-sterile neutrino conversion $\nu_{\alpha} \rightarrow \nu_{s}$ will have to cease when $\mathcal{L}$ approaches zero.

At this point we will be left with grossly non-thermal, non-Fermi-Dirac $\nu_{\alpha}$ and $\nu_{s}$ distributions. Since this process occurs after Weak Decoupling, active neutrino inelastic scattering processes on electrons, nucleons, and 


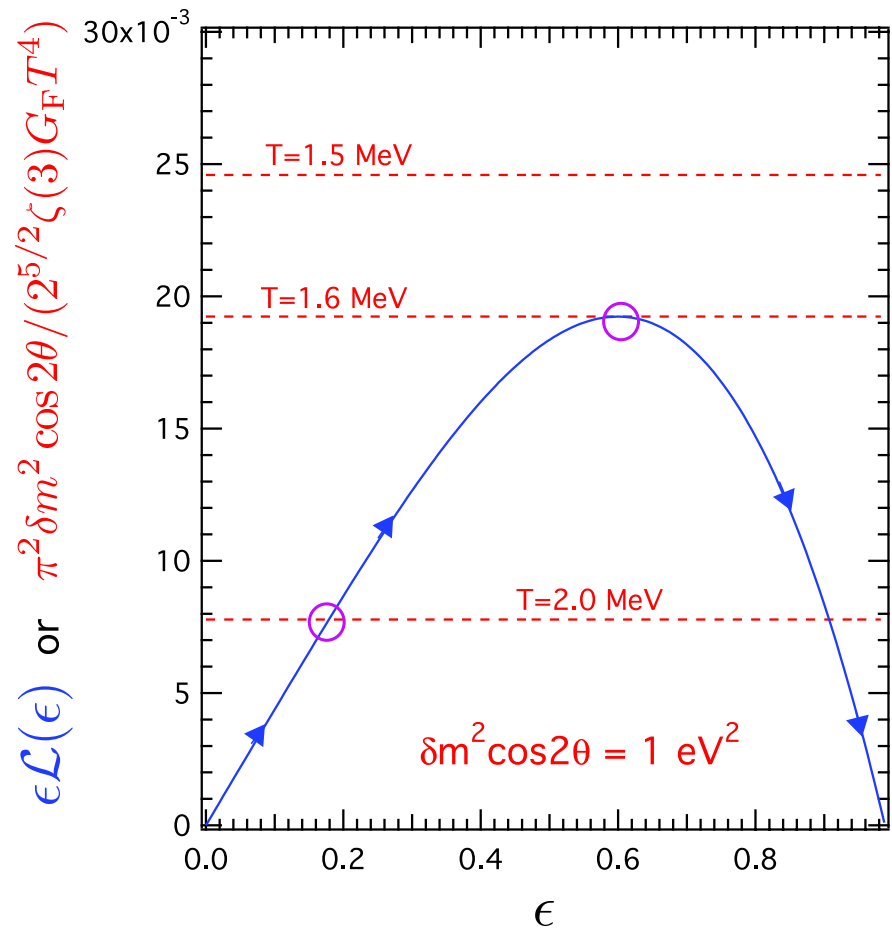

FIG. 2: The solid line is $\epsilon \mathcal{L}(\epsilon)$, the product of $\epsilon$ and potential lepton number, in the smooth and continuous resonance sweep case for initial lepton numbers $L_{\nu_{e}}=L_{\nu_{\mu}}=L_{\nu_{\tau}}=0.01096$, corresponding to initial potential lepton number $\mathcal{L}(\epsilon=0)=$ 0.04384. The arrows give the sense of evolution along this curve as the universe expands and the net potential lepton number decreases as a result of neutrino flavor conversion in the channel $\nu_{e} \rightarrow \nu_{s}$ with $\delta m^{2} \cos 2 \theta=1 \mathrm{eV}^{2}$. The horizontal dashed lines correspond to values of the right-hand side of Eq. 25 for the indicated epochs (temperatures). Solutions to Eq. 25 are possible at a given epoch when the corresponding dashed line crosses the $\epsilon \mathcal{L}(\epsilon)$ curve. Physical solutions are circled here for $T=2.0 \mathrm{MeV}$ and $1.6 \mathrm{MeV}$. The maximum value on the $\epsilon \mathcal{L}(\epsilon)$ curve occurs at $\epsilon_{\max } \approx 0.598$, and in the smooth resonance sweep scenario this is reached at $T \approx 1.6 \mathrm{MeV}$. Clearly, no solutions are possible in this scenario for $T<1.6 \mathrm{MeV}$. If the system were forced to follow the smooth resonance sweep $\epsilon \mathcal{L}(\epsilon)$ curve beyond $\epsilon_{\max }$, the potential lepton number would be completely depleted when $\epsilon$ reaches $\epsilon_{\text {c.o. }} \approx 0.987$ (i.e., $\left.\mathcal{L}\left(\epsilon_{\text {c.o. }}\right)=0\right)$.

other neutrinos have rates which are slow compared to the expansion rate of the universe. This means that these processes will be unable to redistribute effectively the active neutrino occupation numbers and so they cannot morph the $\nu_{\alpha}$ distribution into a thermal distribution. This has consequences for the lepton capture rates on nucleons as we will discuss below in section IV.

In either the (unphysical) smooth and continuous resonance sweep scenario or in some stochastic resonance sweep case both the active neutrino and resulting sterile neutrino distribution functions will be non-thermal in character. Since we cannot solve for resonance sweep beyond $\epsilon_{\max }$ we do not know the final active and sterile neutrino energy spectra. However, for the purposes of

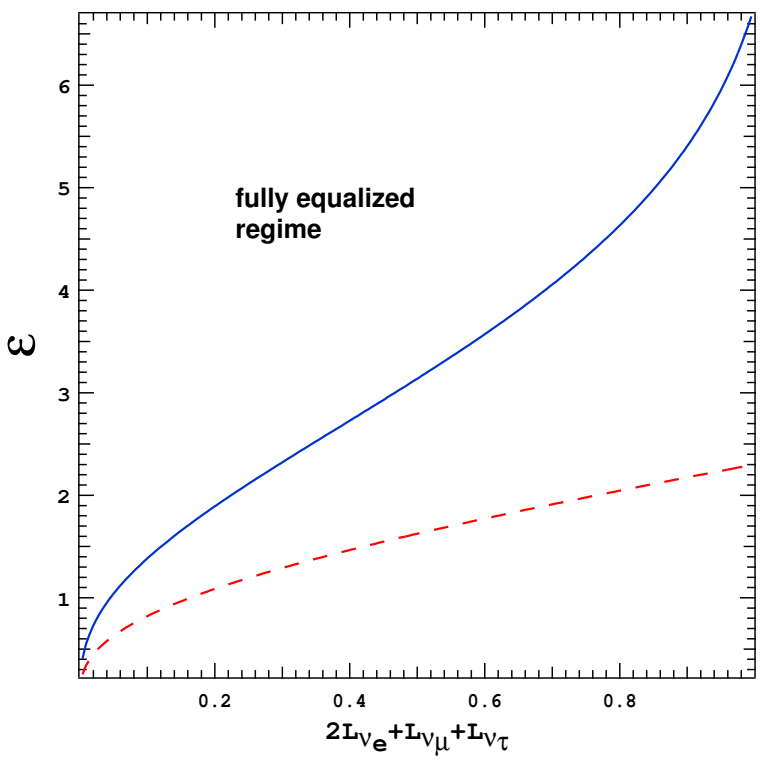

FIG. 3: The values of $\epsilon_{\text {c.o. }}$ (solid line) and $\epsilon_{\max }$ (dashed line) are shown as functions of total initial potential lepton number in the limit of a smooth and continuous resonance sweep and with the assumption that full active neutrino equilibration obtains $\left(L_{\nu_{e}}=L_{\nu_{\mu}}=L_{\nu_{\tau}}\right)$.

constraints and general guidelines, we will find that the idealized smooth and continuous resonance sweep scenario provides the basis for lower limits on the effects of neutrino spectral distortion.

It is useful to consider the solution for the cut-off energy $\epsilon_{\text {c.o. }}$ and the peak energy $\epsilon_{\max }$ in the smooth and continuous resonance sweep case. To get the first of these quantities, we force the system to evolve continuously (e.g., along the solid curve in Fig. 21) all the way to complete lepton number depletion and solve

$$
\mathcal{L}^{\text {initial }}=\frac{3}{4} \frac{1}{F_{2}(0)} \int_{0}^{\epsilon_{\text {c.o. }}} \frac{x^{2}}{e^{x-\eta_{\nu_{\alpha}}+1}} d x,
$$

or $\mathcal{L}\left(\epsilon_{\text {c.o. }}\right)=0$. The second of these quantities is the solution of Eq. (26). Both of these solutions are shown as functions of initial potential lepton number, $\mathcal{L}^{\text {initial }}$ in Fig. (3). In this figure it is assumed that the active neutrinos are fully "equilibrated" initially (before any flavor transformation) with $L_{\nu_{e}}=L_{\nu_{\mu}}=L_{\nu_{\tau}}$.

It is obvious from Eq. (27) that there is a maximum value of the initial potential lepton number for which a solution is obtainable when $\nu_{\alpha} \rightleftharpoons \nu_{s}$ is the only operative neutrino flavor conversion channel. This maximum is given by the limit where $\epsilon_{\text {c.o. }} \rightarrow \infty$,

$$
\mathcal{L}_{\max }^{\text {initial }} \approx \frac{3}{4} \frac{F_{2}\left(\eta_{\nu_{\alpha}}\right)}{F_{2}(0)} .
$$

Scenarios where the bulk of the initial potential lepton number is contained in seas of another flavor of active neutrinos may not allow $\nu_{\alpha} \rightleftharpoons \nu_{s}$ conversion to leave 
a zero final potential lepton number. This is a simple consequence of the post Weak Decoupling conservation of numbers of neutrinos of all kinds. Of course, active-active neutrino flavor transformation in the channels $\nu_{\alpha} \rightleftharpoons \nu_{\beta}$ $(\alpha, \beta=\mathrm{e}, \mu, \tau)$ can alter this picture significantly and will be discussed below.

\section{Efficiency of Neutrino Flavor Conversion: Adiabaticity}

From the previous discussion it is clear that the efficiency of active-sterile neutrino flavor conversion at MSW resonances is a key issue in resolving how these resonances sweep with scaled energy $\epsilon$. This is especially true for values of scaled resonance energy beyond $\epsilon_{\max }$, where we argued that if the resonance condition can be met neutrino flavor transformation was likely to be efficient for the typical neutrino mass/mixing parameters we consider here. It is the adiabaticity of neutrino propagation which determines transformation efficiency both in the active-sterile and active-active channels. We point out here that adiabaticity parameters are high for our chosen epoch and neutrino mass/mixing characteristics, essentially because these parameters are proportional to the ratio of a gravitational time scale (the causal horizon) to a weak timescale (in-medium oscillation time).

The causal horizon (particle horizon) is the proper distance traversed by a null signal in the age of the universe $t$. In radiation-dominated conditions in the early universe this is (setting $c=1$ )

$$
d_{\mathrm{H}}(t)=2 t=H^{-1},
$$

where the local Hubble expansion rate is

$$
H \approx\left(\frac{8 \pi^{3}}{90}\right)^{1 / 2} g^{1 / 2} \frac{T^{2}}{m_{\mathrm{pl}}} .
$$

Here $m_{\mathrm{pl}} \approx 1.221 \times 10^{22} \mathrm{MeV}$ is the Planck mass. The statistical weight for a relativistic boson species $i$ is $\left(g_{b}\right)_{i}$, while that for a relativistic fermion species $j$ is $\left(g_{f}\right)_{j}$. These are related to the total statistical weight $g$ by a sum over all particle species $i$ and $j$ with relativistic kinematics with equilibrium or near equilibrium energy distribution functions and energy densities in the plasma at temperature $T$, given by $g \equiv \sum_{i}\left(g_{b}\right)_{i}+(7 / 8) \sum_{j}\left(g_{f}\right)_{j}$. In the epoch between Weak Decoupling and Weak Freeze Out and BBN, photons, $e^{ \pm}$pairs and the active neutrinos are relativistic and appreciably populated so that $g \approx 10.75$ and $t \approx(0.74 \mathrm{~s})(10.75 / g)^{1 / 2}(\mathrm{MeV} / T)^{2}$. The spectral distortions and extra energy density stemming from the net lepton numbers considered in this paper are usually small effects, causing deviations of the expansion rate from that given above by less than a few percent in many cases.

Homogeneity and isotropy in the early universe imply that the entropy in a co-moving volume is conserved. The proper, physical entropy density in radiation-dominated conditions is $S \approx\left(2 \pi^{2} / 45\right) g_{s} T^{3}$, where $g_{s}$ is closely related to $g$ and we can take $g_{s} \approx g$. We can take the comoving volume element to be the cube of the scale factor $a$ in the Friedman-Lemaitre-Robertson-Walker (FLRW) metric, so that $a^{3} S$ is invariant with FLRW time coordinate $t$ and therefore $g^{1 / 3} a T$ is constant. In turn, this implies that the fractional rate of change of the temperature is related to the expansion rate and the fractional rate of change of the statistical weight by

$$
\frac{\dot{T}}{T} \approx-H\left(1+\frac{\dot{g} / g}{3 H}\right) .
$$

At lower temperatures, where the thermal potential can be neglected, the potential governing neutrino flavor transformation is the difference of the Hamiltonians (e.g., Eq.s (4), (5), (6), (3)) for the transforming neutrino species. For the active-sterile channel $\nu_{\alpha} \rightleftharpoons \nu_{s}$, for example, we have $V \approx H\left(\nu_{\alpha}\right)-H\left(\nu_{s}\right)$. The appropriate potentials for the active-active neutrino flavor transformation channels follow in like manner.

The density scale height for the early universe depends on the neutrino flavor transformation channel and is defined as

$$
\begin{aligned}
\mathcal{H} & \equiv\left|\frac{1}{V} \frac{d V}{d t}\right|^{-1} \\
& \approx \frac{1}{3} H^{-1}\left|1+\frac{\dot{g} / g}{3 H}-\frac{\dot{\mathcal{L}} / \mathcal{L}}{3 H}\right|^{-1} .
\end{aligned}
$$

Here the approximation on the second line is for activesterile neutrino flavor transformation and follows on neglecting the thermal potential $C$ and using Eq. (31). When the statistical weight and the lepton numbers are not changing rapidly the density scale height is roughly a third of the horizon scale. This is $\sim 10^{5} \mathrm{~km}$ at the epoch we are considering here.

Define $\Delta \equiv \delta m^{2} / 2 E_{\nu}$. It can be shown that the ratio of the difference of the squares of the effective masses in matter to twice the neutrino energy is

$$
\begin{aligned}
\Delta_{\mathrm{eff}} & \equiv \frac{\delta m_{\mathrm{eff}}^{2}}{2 E_{\nu}} \\
& \approx \sqrt{(\Delta \cos 2 \theta-V)^{2}+\left(\Delta \sin 2 \theta+B_{e \tau}\right)^{2}},
\end{aligned}
$$

where $\theta$ is the appropriate effective two-by-two vacuum mixing angle and where $V=A+B+C$ is the appropriate potential for the transformation channel. Here $B_{\mathrm{e} \tau}$ is the flavor-off diagonal potential as defined in Qian \& Fuller 1995 26]. The flavor basis off-diagonal potential vanishes, $B_{e \tau}=0$, for any active-sterile mixing channel.

The effective matter (in-medium) mixing angle $\theta_{\mathrm{M}}$ for a neutrino transformation channel with potential $V$ and effective vacuum mixing angle $\theta$ satisfies 


$$
\sin ^{2} 2 \theta_{\mathrm{M}}=\frac{\Delta^{2} \sin ^{2} 2 \theta\left(1+2 E_{\nu} B_{e \tau} / \delta m^{2} \sin 2 \theta\right)^{2}}{(\Delta \cos 2 \theta-V)^{2}+\Delta^{2} \sin ^{2} 2 \theta\left(1+2 E_{\nu} B_{e \tau} / \delta m^{2} \sin 2 \theta\right)^{2}} .
$$

The effective matter mixing angle for the antineutrinos in this channel, $\bar{\theta}_{\mathrm{M}}$, satisfies a an expression which has opposite signs for the potentials $B, A$, and $B_{e \tau}$, but which is otherwise identical.

The change in the potential required to drop the effective matter mixing from the maximal resonant value $\left(\theta_{\mathrm{M}}=\pi / 4\right)$ to a value where $\sin ^{2} 2 \theta_{\mathrm{M}}=1 / 2$ is termed the resonance width and is

$$
\delta V \approx \Delta \sin 2 \theta\left|1+\frac{2 E_{\nu} B_{e \tau}}{\delta m^{2} \sin 2 \theta}\right| .
$$

The physical width in space, or in FLRW coordinate time $t$, corresponding to this potential width is

$$
\begin{aligned}
\delta t & =\left.\frac{d t}{d V} \delta V \approx\left|\frac{1}{V} \frac{d V}{d t}\right|^{-1} \frac{\delta V}{V}\right|_{\text {res }} \\
& \approx \mathcal{H} \tan 2 \theta\left|1+\frac{2 E_{\nu} B_{e \tau}}{\delta m^{2} \sin 2 \theta}\right| .
\end{aligned}
$$

The local neutrino oscillation length at resonance is

$$
L_{\mathrm{osc}}^{\mathrm{res}}=\frac{4 \pi E_{\nu}}{\delta m_{\mathrm{eff}}^{2}}=\frac{2 \pi}{\Delta_{\mathrm{eff}}} \approx \frac{2 \pi}{\delta V},
$$

where the latter approximation is good only at resonance. We can define the dimensionless adiabaticity parameter as proportional to the ratio of the resonance width and the neutrino oscillation length at resonance:

$$
\begin{aligned}
\gamma & \equiv 2 \pi \frac{\delta t}{L_{\mathrm{osc}}^{\mathrm{res}}} \approx \delta t \delta V \\
& \approx \frac{\delta m^{2} \mathcal{H}}{2 E_{\nu}} \cdot \frac{\sin ^{2} 2 \theta}{\cos 2 \theta} \cdot\left|1+\frac{2 E_{\nu} B_{e \tau}}{\delta m^{2} \sin 2 \theta}\right|^{2} .
\end{aligned}
$$

This parameter can be evaluated anywhere in the evolution of neutrino flavors, even well away from resonances and it will serve to gauge the degree to which neutrinos tend to remain in mass eigenstates. The Landau-Zener jump probability, assuming a linear change in potential across the resonance width, is $P_{\mathrm{LZ}} \approx \exp (-\pi \gamma / 2)$, so that it is clear that a large value of the adiabaticity parameter corresponds to a small probability of jumping between mass eigenstate tracks and, hence, efficient flavor conversion at asymptotically large distance (many resonance widths) from resonance.

Folding in the expansion rate in radiation-dominated conditions, using the conservation of co-moving entropy density, and assuming that we can neglect the thermal potential $C$, we can show that the adiabaticity parameter for neutrino propagation through an active-sterile resonance is

$$
\begin{aligned}
\gamma & \approx \frac{\sqrt{5} \zeta^{3 / 4}(3)}{2^{1 / 8} \pi^{3}} \cdot \frac{\left(\delta m^{2}\right)^{1 / 4} m_{\mathrm{pl}} G_{\mathrm{F}}^{3 / 4}}{g^{1 / 2}} \cdot\left[\frac{\mathcal{L}^{3 / 4}}{\epsilon^{1 / 4}}\right] \cdot\left[\frac{\sin ^{2} 2 \theta}{\cos ^{7 / 4} 2 \theta}\right] \cdot\left|1+\frac{\dot{g} / g}{3 H}-\frac{\dot{\mathcal{L}} / \mathcal{L}}{3 H}\right|^{-1} \\
& \approx\left(\frac{10.75}{g}\right)^{1 / 2} \cdot\left[\frac{\delta m^{2}}{1 \mathrm{eV}^{2}}\right]^{1 / 4} \cdot \frac{1}{\epsilon^{1 / 4}} \cdot\left[\frac{\mathcal{L}}{0.01}\right]^{3 / 4} \cdot\left|1+\frac{\dot{g} / g}{3 H}-\frac{\dot{\mathcal{L}} / \mathcal{L}}{3 H}\right|^{-1} \cdot\left\{\frac{\sin ^{2} 2 \theta}{1.77 \times 10^{-8}}\right\}
\end{aligned}
$$

In these expressions $\epsilon=E_{\nu} / T$ is the scaled energy of a neutrino at resonance in a channel $\nu_{\alpha} \rightleftharpoons \nu_{s}$ characterized by the difference of the squares of the appropriate vacuum mass eigenvalues, $\delta m^{2}$. It is obvious from these considerations that neutrino flavor transformation will be efficient at resonance (i.e., $\gamma \gg 1$ ) over broad ranges of energy for the regime of the early universe between Weak Decoupling and Weak Freeze Out even for very small effective vacuum mixing angle $\theta$.

Eq. (39) shows that two trends can eventually destroy adiabaticity and, therefore, large scale resonant activesterile neutrino flavor transformation. As active neutri- nos are converted $\mathcal{L}$ is reduced and this reduces $\gamma$. In turn, the fractional rate of destruction of $\mathcal{L}$ compared with the Hubble parameter can be become significant, especially if $\mathcal{L}$ is small, and this can also reduce $\gamma$.

\section{Active-active Neutrino Flavor Conversion and Equilibration}

Active neutrinos $\left(\nu_{e}, \bar{\nu}_{e}, \nu_{\mu}, \bar{\nu}_{\mu}, \nu_{\tau}, \bar{\nu}_{\tau}\right)$ transforming among themselves on time scales comparable to or shorter than that of the active-sterile conversion chan- 


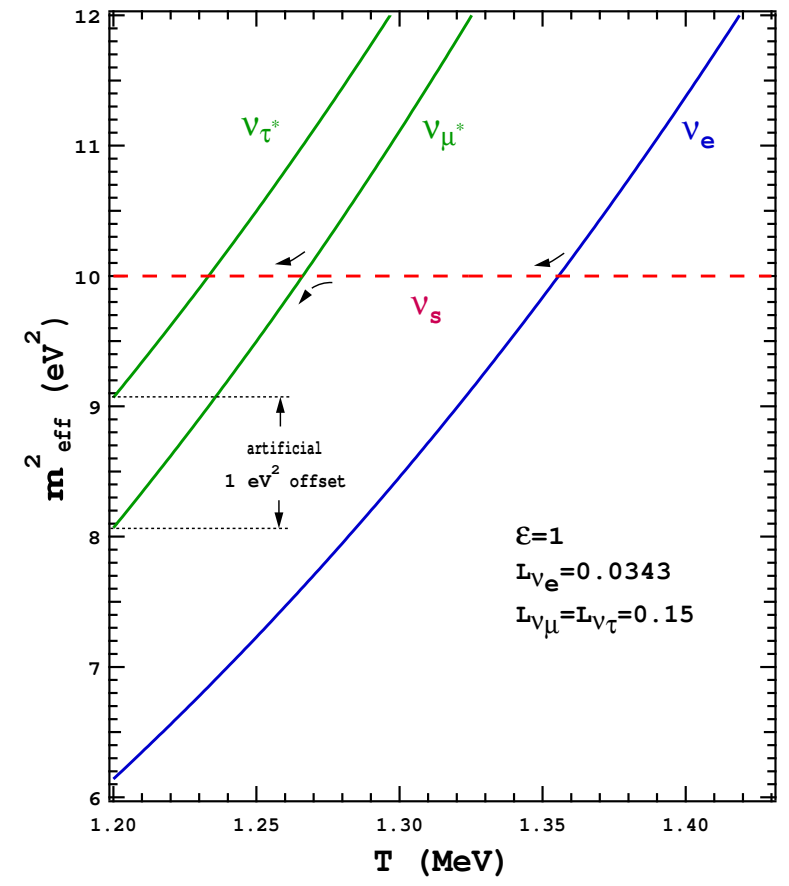

FIG. 4: Level crossing diagram for the case with lepton numbers as shown and for scaled neutrino energy $\epsilon=1$. The vacuum mass-squared eigenvalue for the (mostly) sterile state is taken as $m_{4}^{2}=10 \mathrm{eV}^{2}$. This is shown as the dashed curve labeled $\nu_{s}$. An artificial (exaggerated) $1 \mathrm{eV}^{2}$ offset between the vacuum mass-squared eigenvalues $m_{2}^{2}$ and $m_{3}^{2}$ has been added so that the curves labeled with $\nu_{\mu}^{*}$ and $\nu_{\tau}^{*}$ are separated for clarity. In reality, the top curve should be split from the lower curve by $\delta m^{2} \approx 3 \times 10^{-3} \mathrm{eV}^{2}$. Conversion in the channel $\nu_{e} \rightarrow \nu_{s}$ is as described in the text.

nel can alter significantly the scenario for sterile neutrino production given above. This is apt to be the case if active-active neutrino mixing in medium is large and efficient over a broad range of neutrino energies. Activesterile neutrino flavor conversion tends to be slow because it occurs through MSW resonances and the rate at which these resonances sweep through the neutrino distribution functions is determined by the expansion of the universe, a slow gravitational time scale.

Coherent neutrino flavor conversion in active-active channels in the early universe can be dominated by the flavor off-diagonal potential. Large in-medium mixing angles can accompany the synchronization seen in calculations of active-active mixing in supernovae and the early universe [17]. If active-active neutrino flavor transformation is efficient, then lepton numbers in different active neutrino species can be quickly equilibrated, meaning instantaneous equal lepton numbers.

The flavor diagonal neutrino forward scattering potential in an active-active channel $\nu_{\alpha} \rightleftharpoons \nu_{\beta}$ is $A+B=$ $H\left(\nu_{\alpha}\right)-H\left(\nu_{\beta}\right)$. If there is an initial disparity in lepton number in these two flavors then matter-enhanced or -suppressed transformation will go in the direction of reducing this disparity. Though initially the flavor off- diagonal potential $B_{e \tau} \approx 0$, as soon as flavor transformation begins this potential comes up.

The interplay of matter-enhanced coupled activesterile and active-active neutrino flavor transformation can be complicated and difficult to follow numerically. The size of the debit in the $\nu_{e}$ or $\bar{\nu}_{e}$ distributions (e.g., the final value of $\epsilon_{\text {c.o. }}$ or $\epsilon_{\max }$ in a continuous sweep scenario) may be much more complicated in the general $4 \times 4$ case than the scenario outlined above for "simple" $2 \times 2$ $\nu_{\alpha} \rightleftharpoons \nu_{s}$ interconversion. We can, however, identify a few cases where we can at least outline the course of neutrino flavor conversion as the universe expands and cools. We will therefore consider two limits: (1) no active-active mixing; and (2) efficient active-active mixing that guarantees that lepton numbers in active species are always the same (instantaneous equilibration).

\section{E. Inefficient Active-Active Neutrino Flavor Conversion}

Consider first the case where we neglect active-active neutrino mixing effects. In this case we could have initial lepton numbers that are not fully equilibrated. For example, we could have a scenario where initially $L_{\nu_{e}}<L_{\nu_{\mu}}=L_{\nu_{\tau}}$. In this case the $\nu_{\mu}$ and $\nu_{\tau}$ experience the largest effective potentials, and hence have the largest effective masses at a given temperature (epoch) in the early universe. Therefore, the first (highest temperature) resonance occurs for $\nu_{s}$ with $\nu_{e}$, as illustrated in Fig. (41). This resonance will destroy lepton number, as will the subsequent $\nu_{\mu}^{*} \rightleftharpoons \nu_{s}$ resonance, and it will leave a distorted $\nu_{e}$ spectrum.

Here we follow Ref. 27] and define linear combinations of the muon and tauon neutrino flavor states

$$
\begin{aligned}
\left|\nu_{\mu}^{*}\right\rangle & \equiv \frac{\left|\nu_{\mu}\right\rangle-\left|\nu_{\tau}\right\rangle}{\sqrt{2}} \\
\left|\nu_{\tau}^{*}\right\rangle & \equiv \frac{\left|\nu_{\mu}\right\rangle+\left|\nu_{\tau}\right\rangle}{\sqrt{2}} .
\end{aligned}
$$

This reduces the $4 \times 4$ mixing problem of three active neutrinos and a sterile neutrino into a $3 \times 3$ problem with $\left|\nu_{\tau}^{*}\right\rangle$ decoupled (a mass eigenstate in vacuum with no mixing with the other neutrinos). This reduction in dimensionality of the neutrino mixing problem works in vacuum only if the muon and tauon neutrinos are maximally mixed. It will be valid in medium only if, additionally, these two neutrino flavors experience identical matter interactions. This latter condition is met if $L_{\nu_{\mu}}=L_{\nu_{\tau}}$. This symmetry condition will be respected so long as muon and tauon neutrinos behave and transform identically. Indeed, the second resonance encountered as the universe cools, $\nu_{\mu}^{*} \rightleftharpoons \nu_{s}$, respects this condition as the $\left|\nu_{\mu}^{*}\right\rangle$ state consists of equal parts muon and tauon states.

The sterile neutrinos produced through the $\nu_{e} \rightarrow \nu_{s}$ resonance are subsequently transformed into $\nu_{\mu}^{*}$ at the second resonance at lower temperature, as depicted for a particular set of initial lepton numbers in Fig. (4). This 
resonance also converts the $\nu_{\mu}^{*}$ into the sterile state, so that the final abundance of sterile neutrinos results from the conversion of neutrinos which were originally in the $\nu_{\mu}$ and $\nu_{\tau}$ distributions. Since these distributions have higher lepton number than resides in the $\nu_{e} / \bar{\nu}_{e}$ seas, the final number density of sterile neutrinos will be larger than the number of $\nu_{e}$ missing from the $\nu_{e}$-distribution. As we will see, this case may be more likely to be in conflict with massive neutrino dark matter constraints.

If we temporarily ignore the effect of active-active neutrino flavor transformations, then we can make some general statements about the change in the lepton numbers and $\epsilon$ for the $\nu_{e}$ or $\bar{\nu}_{e}$ distributions in this case of unequal $L_{\nu_{e}}$ and $L_{\nu_{\mu}}=L_{\nu_{\tau}}$. If the potential lepton number for electron flavor neutrinos $\mathcal{L}_{e}$ is driven to zero first then the changes in the individual active neutrino lepton numbers must be related by

$$
2 \Delta L_{\nu_{e}}+\Delta L_{\nu_{\mu}^{*}}=-\mathcal{L}_{e}^{\text {initial }}
$$

where here $L_{\nu_{\mu}^{*}}=\left(L_{\nu_{\mu}}+L_{\nu_{\tau}}\right) / 2$, and $\Delta L_{\nu_{e}}=\Delta n_{\nu_{e}} / n_{\gamma}$ and $\Delta L_{\nu_{\mu}}=\Delta n_{\nu_{\mu}} / n_{\gamma}$.

Of course, if $\mathcal{L}_{e}$ is driven to zero before $\mathcal{L}_{\mu}^{*}$, then conversion of $\nu_{e}$ 's at the first resonance will cease while conversion in the channel $\nu_{\mu}^{*} \rightarrow \nu_{s}$ continues until $\mathcal{L}_{\mu^{*}}$ is reduced to zero. This will leave $\mathcal{L}_{e}<0$ which will result in anti-electron neutrino transformation $\bar{\nu}_{e} \rightarrow \bar{\nu}_{s}$, leaving a non-thermal deficit in the $\bar{\nu}_{e}$ distribution.

This is likely temporary, however. The $\nu_{e}$ potential is zero at the point where $\mathcal{L}_{e}$ first vanishes. Thereafter, with reduction in $\mathcal{L}_{\mu^{*}}$, the $\nu_{e}$ potential's magnitude first increases, but then decreases as the universe expands and the temperature drops. This can be seen from Eq. 14 and on noting that the potential behaves like $V \sim \mathcal{L}_{e} T^{3}$. The evolution of the potential with time is determined by the competition between two effects. The conversion of lepton number discussed above makes $\mathcal{L}_{e}$ more negative and larger in magnitude, while the expansion of the universe decreases $T$. Therefore, the antineutrinos could experience two resonances: (1) first when $\mathcal{L}_{e}$ becomes sufficiently negative that the potential $V$ becomes large enough in magnitude to satisfy Eq. (14); and (2) subsequently when the temperature drops enough that this condition is again satisfied. At the first resonance we have $\bar{\nu}_{e} \rightarrow \bar{\nu}_{s}$, but these steriles are reconverted at the second resonance, $\bar{\nu}_{s} \rightarrow \bar{\nu}_{e}$. This can also be viewed from the perspective of resonance sweep. Note that $\epsilon_{\text {res }}$ is infinite when $\mathcal{L}_{e}$ first crosses zero, but then decreases as $\mathcal{L}_{e}$ becomes larger in magnitude as lepton number is converted, but then "turns around" and begins to sweep toward higher energy again once $T$ becomes low enough. This process is directly analogous to the re-conversion of $\bar{\nu}_{s}$ neutrinos in neutrino-heated outflow in supernovae [28].

In this scenario it is the mu and tau neutrinos, ultimately, that are converted to sterile neutrinos so that the numbers and kinds of converted active neutrinos are given by

$$
\mathcal{L}_{\mu^{*}}^{\text {init }} \approx \frac{2}{n_{\gamma}}\left(\Delta n_{\nu_{\mu}^{*}}+\Delta n_{\nu_{\mu}^{*}}^{\prime}\right)-\frac{1}{n_{\gamma}}\left(\Delta n_{\nu_{e}}+\Delta n_{\bar{\nu}_{e}}\right),
$$

where $\Delta n_{\nu_{\mu}^{*}}$ and $\Delta n_{\nu_{\mu}^{*}}^{\prime}$ are the number of $\nu_{\mu}^{*}$ neutrinos converted before and after $\mathcal{L}_{e}$ first vanishes, respectively. Likewise, $\Delta n_{\nu_{e}}$ electron neutrinos are converted before $\mathcal{L}_{e}$ first vanishes and $\Delta n_{\bar{\nu}_{e}}$ electron antineutrinos afterward, though these $\bar{\nu}_{e}$ 's are eventually returned to the distribution.

There is an additional complication: in the case that all three lepton numbers are equal, the mass-squared differences between the active states are approximately given by their vacuum values, which are quite small. The three resonances depicted in Fig. (4) will then be very close together, and in fact may overlap if the resonance width is sizable.

If the resonances do not overlap, the lepton number destroying resonance will take place between $\nu_{1}$ and $\nu_{s}$, where $\nu_{1}$ is the lightest neutrino mass eigenstate. Since $\nu_{1}$ has a large $\nu_{e}$ component, this will leave a non-thermal $\nu_{e}$ distribution, and in addition there will be smaller nonthermal distortions of the $\nu_{\mu}$ and $\nu_{\tau}$ spectra. In the case that the resonances do overlap, the full details of the evolution will be quite complicated, but a similar outcome is obtained nonetheless. To summarize, in all cases a non thermal $\nu_{e}$ spectrum results.

\section{F. Efficient Active-Active Mixing: Instantaneously Equilibrated Lepton Numbers}

Let us now consider the limit where in addition to the active-sterile MSW transitions, oscillations/transformations between/among the three active neutrinos occur simultaneously and are efficient. If active-active mixing among all the active flavors is instantaneous and efficient then we only need to consider the case where the lepton numbers are equal, $L_{e}=L_{\mu}=L_{\tau}$, both initially and as active-sterile transformation proceeds. It has been shown that large angle mixing between the three active neutrino species results in the system being driven toward such an equilibrated state [17] at a temperature of $T \gtrsim 2 \mathrm{MeV}$.

An obvious additional effect of efficient active-active oscillations will be to partially refill any hole that was left in the $\nu_{e}$ distribution. It is important to note, though, that this refilling cannot be complete. For maximal $\nu_{e}-\nu_{\mu, \tau}$ mixing, the hole in the distribution can be only partially refilled. In vacuum the measured solar neutrino mixing angle is less than maximal, $\theta_{\text {solar }} \simeq 32.5^{\circ}$, and $U_{e 3}$ is relatively small. In medium, at best we will obtain maximal matter mixing angles in the limit where the flavor off-diagonal potential is large. We again therefore expect about 0 to $2 / 3$ refilling at most, so that a nonthermal $\nu_{e}$ spectrum is always obtained by the epoch of Weak Freeze Out. Even if it were somehow possible for the resonance to effectively involve only $\nu_{s}$ and $\nu_{\mu} / \nu_{\tau}$, 


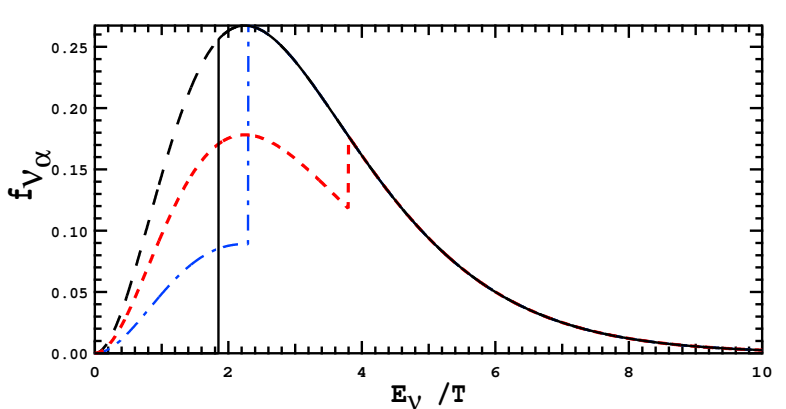

FIG. 5: Final active neutrino energy distribution function $f_{\nu_{\alpha}}$ for Cases 1 (short dashed line), 2 (dot dashed line), and 3 (solid line) for the particular case of a continuous resonance sweep scenario and in the instantaneous active-active mixing limit as described in the text. Here $\alpha=\mathrm{e}, \mu, \tau$ : all species have the same distribution function. The long dashed line and its continuation as a solid line shows the original thermal distribution function common to all active flavors. The particular scenario shown here has $L_{\nu_{e}}=L_{\nu_{\tau}}=L_{\nu_{\mu}}=0.1$, so that $\epsilon_{1} \approx 3.8, \epsilon_{2} \approx 2.3$, and $\epsilon_{3} \approx 1.85$

active-active oscillations would again act to refill the hole in the resulting non thermal $\nu_{\mu}$ and/or $\nu_{\tau}$ spectra, and in so doing create a non-thermal $\nu_{e}$ distribution.

We can identify three cases.

Case 1: We have only one sterile neutrino species and only one channel for its production, $\nu_{\alpha} \rightarrow \nu_{s}$. If this channel is, e.g., $\nu_{e} \rightarrow \nu_{s}$, then the neutrinos in the $\nu_{\tau}$ and $\nu_{\mu}$ distributions will, in the limit of instantaneous maximal mixing, partially fill in the hole left by the activesterile conversion process. Given the boundary condition of equal lepton numbers in all active flavors at all times, a continuous smooth resonance sweep scenario will leave each active neutrino distribution with a low energy "hole" with $2 / 3$ of the normal population out to some value of scaled neutrino energy $\epsilon_{1}$. In terms of the initial potential lepton number $\mathcal{L}^{\text {init }}=\mathcal{L}_{e}=\mathcal{L}_{\mu}=\mathcal{L}_{\tau}$, this is obtained by solving the integral equation

$$
2 \mathcal{L}^{\text {init }} \approx \frac{1}{F_{2}(0)} \int_{0}^{\epsilon_{1}} \frac{x^{2} d x}{e^{x-\eta}+1}
$$

Of course, the continuous resonance sweep scenario is an unphysical idealization and the actual energy spectra will likely be far more complicated, as argued above. However, no matter the resonance sweep physics, in this instantaneous mixing limit the numbers (number densities) of active neutrinos in each flavor are equal and their energy spectra are identical. In the continuous resonance sweep scenario, in the scaled energy interval 0 to $\epsilon_{1}$, the deficit of neutrinos, $\Delta n_{\nu}$, is $1-2 / 3=1 / 3$ of the original population and this deficit is identical for each active flavor. The entire original population of one of the active neutrino flavors in this scaled energy interval is converted to sterile neutrinos so the number density of steriles will be $n_{s}=3 \Delta n_{\nu}$ and this is related to the initial potential lepton number through

$$
\frac{n_{s}}{n_{\gamma}}=\frac{3 \Delta n_{\nu}}{n_{\gamma}}=\frac{3}{4} \mathcal{L}^{\text {init }} .
$$

If there is one light sterile neutrino, there may be others. In fact it has been claimed that two sterile species are a better fit to the LSND data than just one 29]. So this suggests Cases 2 and 3.

Case 2: Allow two channels of sterile neutrino production and two kinds of light sterile neutrinos $\nu_{s 1}$ and $\nu_{s 2}$. As an example, we could have $\nu_{e} \rightarrow \nu_{s 1}$ and $\nu_{\mu} \rightarrow \nu_{s 2}$, but again with instantaneous mixing among all the active neutrino flavors. A continuous resonance sweep scenario will leave each active neutrino distribution with a low energy hole now with $1 / 3$ of the normal population out to some value of scaled neutrino energy $\epsilon_{2}$. In terms of the initial potential lepton number $\mathcal{L}^{\text {init }}=\mathcal{L}_{e}=\mathcal{L}_{\mu}=\mathcal{L}_{\tau}$, this is obtained by solving the integral equation

$$
\mathcal{L}^{\text {init }} \approx \frac{1}{F_{2}(0)} \int_{0}^{\epsilon_{2}} \frac{x^{2} d x}{e^{x-\eta}+1} .
$$

Again, the numbers of active neutrinos in each flavor in scaled energy interval 0 to $\epsilon_{2}$ are equal and so are the deficits, $\Delta n_{\nu}$, which are now $2 / 3$ of the original population in this interval. Now, however, the original populations of two active flavors in this interval are converted to sterile species so the total number density of sterile neutrinos of all kinds is $n_{s}=2 \cdot(3 / 2) \Delta n_{\nu}=3 \Delta n_{\nu}$ and we have the same relation between total sterile neutrino number density $n_{s}$, deficit per flavor $\Delta n_{\nu}$, and initial potential lepton number as in Eq. (45).

For a given initial potential lepton number, this is the same total number of sterile neutrinos (of all kinds) produced as in Case 1. However, since there are now two channels for $\nu_{s}$ production, $\epsilon_{2}$ is smaller than $\epsilon_{1}$. In Case $1, \epsilon_{1}$ is relatively larger because as $\nu_{e}$ 's are converted to steriles two active neutrino distributions compensate by feeding neutrinos into the hole, forcing the resonance to sweep further (higher in energy) through the $\nu_{e}$ distribution to erase the net lepton numbers. In Case 2 only one active neutrino distribution remains to compensate for the hole.

Case 3: Allow all three active neutrinos to convert simultaneously to three kinds of light sterile neutrinos $\nu_{s 1}, \nu_{s 2}, \nu_{s 3}$. Now a smooth resonance sweep scenario will leave each active neutrino distribution with a low energy hole with zero population out to some value of scaled neutrino energy $\epsilon_{3}$. In terms of the initial potential lepton number $\mathcal{L}^{\text {init }}=\mathcal{L}_{e}=\mathcal{L}_{\mu}=\mathcal{L}_{\tau}$, this is obtained by solving the integral equation

$$
\frac{2}{3} \mathcal{L}^{\text {init }} \approx \frac{1}{F_{2}(0)} \int_{0}^{\epsilon_{3}} \frac{x^{2} d x}{e^{x-\eta}+1} .
$$

Now the deficits $\Delta n_{\nu}$ in each active flavor are equal to the original active neutrino populations in the scaled energy interval 0 to $\epsilon_{3}$. Since all three active species transform 
to sterile neutrinos, the total number density of steriles of all kinds is $n_{s}=3 \Delta n_{\nu}$. The relation between $n_{s}$, $\Delta n_{\nu}$ and the initial potential lepton number is the same as in Eq. (45). For a given $\mathcal{L}^{\text {init }}$ this is the same total number of sterile neutrinos produced as in Cases 1 and 2. However, our three cases will have $\epsilon_{1}>\epsilon_{2}>\epsilon_{3}$ for a given initial potential lepton number, for the reasons indicated in the last paragraph.

For Cases 1, 2, and 3 the active neutrino distribution functions will be left with population deficits relative to the thermal case. This is shown in Fig. (5) for the particular scenario where each active flavor starts out with lepton number $L_{\nu_{e}}=L_{\nu_{\tau}}=L_{\nu_{\mu}}=0.1$. Solving the above equations for the three cases yields $\epsilon_{1} \approx 3.8, \epsilon_{2} \approx 2.3$, and $\epsilon_{3} \approx 1.85$ in this example.

In obvious fashion all of the above discussion applies to $\bar{\nu}_{s}$ production if the initial lepton numbers are negative. We should also note that the actual active and sterile neutrino energy distributions in all of the limits considered here may differ considerably from those shown in the figures. This is partly because the continuous resonance sweep cannot continue to completion as described in the last section but may skip to higher scaled energy discontinuously. However, another source of difference from the simple spectra shown in the figures may be because multiple neutrino mixing can be complicated.

We have here presented a picture where the sterile neutrino undergoes a resonance with one of the active neutrino flavors, $\nu_{\alpha}$. However, if the initial lepton numbers are equal, the resonance will instead occur between $\nu_{s}$ and a superposition of the three active neutrinos. In principle, all three of the active neutrinos may mix with the sterile, so the MSW resonance which is responsible for lepton number destruction may occur for the sterile neutrino and a linear superposition of the three active neutrinos. For example, in the so called " $3+1$ " LSND inspired mixing scheme, both $\theta_{14}$ and $\theta_{24}$ are required to be non-zero, and the sterile effectively mixes with all three active neutrinos (as there will also be indirect $\nu_{\tau}-\nu_{s}$ mixing).

In any case, post-decoupling neutrino mixing cannot completely undo spectral distortions. We conclude that a sterile neutrino in the mass range of interest is almost certain to leave non-thermal active neutrino distribution functions if the lepton number is significant.

\section{CONSTRAINTS ON STERILE NEUTRINOS AND LEPTON NUMBERS}

The entire plausible range of sterile neutrino masses and net lepton numbers of interest is not likely to be consistent with all of the current observational bounds. For example, we may demand that the initial net lepton numbers are large enough to suppress the production of fully thermalized seas of $\nu_{s}$ and $\bar{\nu}_{s}$. Eq. (2) shows that the lepton number necessary for suppression of thermal sterile neutrino production depends both on neutrino mass and neutrino average energy. We will hold off on considering BBN effects/limits until the next section.

As discussed in the introduction, a population of sterile neutrinos could provide a sufficiently large contribution to the dark matter density, depending again on sterile neutrino mass, to run afoul of large scale structure/CMB bounds [8]. The above-cited analysis of the SDSS data [10] using CMB anisotropy limits, galaxy clustering and bias, and coupled with the matter power spectrum inferred from the Lyman-alpha forest suggest a limit on the neutrino mass of $0.79 \mathrm{eV}(95 \% \mathrm{CL})$. This corresponds to a limit on the neutrino closure fraction

$$
\Omega_{\nu}^{\lim } h^{2}<0.0084(95 \% \mathrm{CL})
$$

where $h$ is the Hubble parameter at the current epoch in units of $100 \mathrm{~km} \mathrm{~s}^{-1} \mathrm{Mpc}^{-1}$. This is comparable to the older Wilkinson Microwave Anisotropy Probe (WMAP) bound, $\Omega_{\nu}^{\lim } h^{2}<0.0076$ (95\% CL). However, the Eq. (48) bound is more appropriate here as it assumes a " $3+1$ " neutrino mass scenario in contrast to the three neutrinos with a common mass assumed in the WMAP analysis. Of course, this mass limit and our inferred limit on the closure fraction are approximate because our sterile neutrinos have nonthermal energy spectra. Adopting the Eq. (48) bound suggests that thermal distributions of $\nu_{\alpha}$ and $\bar{\nu}_{\alpha}$ neutrinos are acceptable only if they have rest masses

$$
m_{\nu_{\alpha}} \lesssim 0.79 \mathrm{eV}\left[\frac{2 F_{2}(0)}{F_{2}\left(\eta_{\nu_{\alpha}}\right)+F_{2}\left(-\eta_{\nu_{\alpha}}\right)}\right]\left[\frac{\Omega_{\nu}^{\lim } h^{2}}{0.0084}\right],
$$

where $\alpha=\mathrm{e}, \mu, \tau, \mathrm{s}$. We can connect this with the a putative thermal sterile neutrino sea by noting that $m_{\nu_{s}} \approx\left(\delta m_{\text {as }}^{2}\right)^{1 / 2}$. So, for example, $\delta m_{\text {as }}^{2}>0.63 \mathrm{eV}^{2}$ is disallowed if all the sterile neutrino species have thermal distributions. This would eliminate much of the LSNDinspired sterile neutrino mass range.

However, the coherent sterile neutrino production scenarios discussed above may do better at creeping in under the closure contribution bound. For one thing, only $\nu_{s}\left(\right.$ or $\left.\bar{\nu}_{s}\right)$ and not its opposite helicity partner are produced coherently. Furthermore, the sterile neutrinos are produced in numbers of order the initial lepton number. This will be smaller than a general thermal population.

At the epoch of coherent sterile neutrino production the ratio of the number of active neutrinos $\Delta n_{\nu_{\alpha}}$ converted to steriles to the number total density of a thermal distribution of $\nu_{\alpha}$ plus $\bar{\nu}_{\alpha}$ neutrinos is in the ratio of the closure contributions of a sterile species to thermal neutrino species:

$$
\frac{\Omega_{s} h^{2}}{\Omega_{\nu_{\alpha}+\bar{\nu}_{\alpha}}^{\text {therm }} h^{2}} \approx R_{s} \equiv \frac{N_{s} \Delta n_{\nu_{\alpha}}}{n_{\nu_{\alpha}}+n_{\bar{\nu}_{\alpha}}}
$$

where $N_{s}$ is the number of active-sterile mixing channels operating in the production of sterile neutrinos. In turn it can be shown that in the continuous resonance sweep 


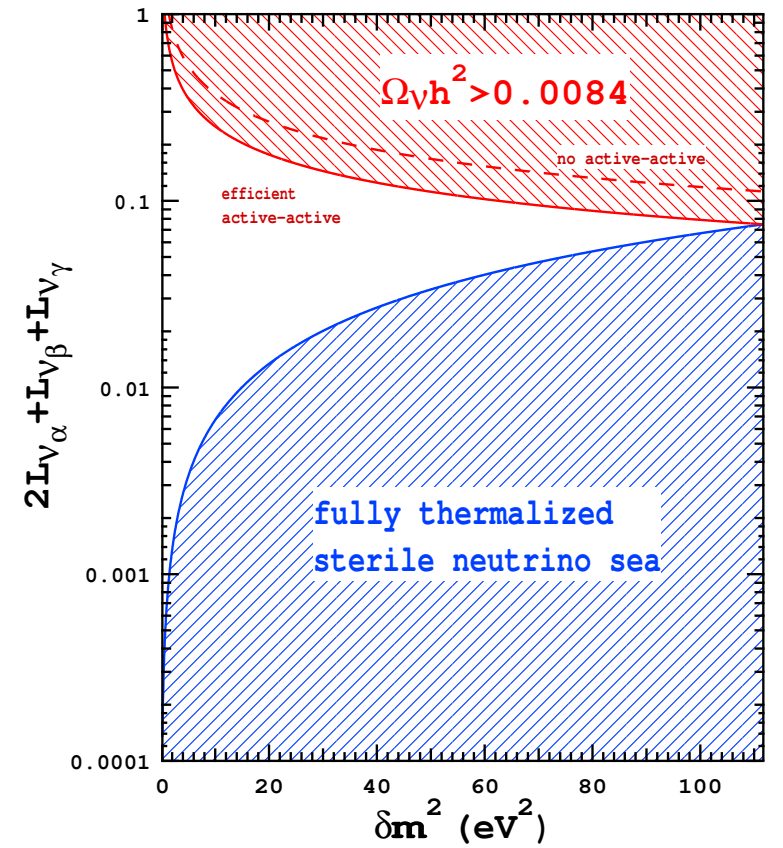

FIG. 6: Constraints on the the ranges of active-sterile masssquared splitting and potential lepton number as derived in the smooth and continuous resonance sweep limit. Parameter ranges which give sufficient sterile neutrino production to exceed the bound on the neutrino closure fraction are shown cross hatched, as are parameter ranges which allow for complete or nearly complete thermal, undiluted energy distribution functions for a sterile species. The upper solid line is for efficient active-active mixing in Cases 1,2 , or 3 , while the upper dashed line gives the constraint for $\nu_{\alpha} \rightarrow \nu_{s}$ with no active-active mixing.

scenario

$$
R_{s} \approx\left[\frac{1}{F_{2}(0)} \int_{0}^{\epsilon_{\text {c.o. }}} \frac{x^{2} d x}{e^{x-\eta}+1}\right]\left[\frac{F_{2}(0)}{F_{2}\left(\eta_{\nu_{\alpha}}\right)+F_{2}\left(-\eta_{\nu_{\alpha}}\right)}\right]
$$

where $\epsilon_{\text {c.o. }}$ and the degeneracy parameter $\eta$ are values consistent with the particular sterile neutrino production scheme. From these relations we can show that

$$
\Omega_{s} h^{2} \approx\left(1.062 \times 10^{-2}\right)\left(\frac{\beta}{2}\right) \mathcal{L}\left[\frac{\delta m_{\mathrm{as}}^{2}}{\mathrm{eV}^{2}}\right]^{1 / 2}
$$

where $\mathcal{L}$ is an appropriate potential lepton number and where $\beta$ is a parameter that is related to the particular sterile neutrino production scheme and the number of active-sterile channels in that scheme. For example, $\beta=2$ for Cases 1,2 , and 3 of the efficient active-active limit, whereas $\beta=4 / 3$ for $\nu_{\alpha} \rightarrow \nu_{s}$ only with no activeactive mixing. All of these constraints are summarized in Fig. (6).

Though we have employed the continuous resonance sweep scenario, the constraints shown stem from closure fraction considerations and, therefore, depend principally on numbers of sterile neutrinos produced and on their rest mass. The number of sterile neutrinos is tied to the initial potential lepton number and is insensitive to the details of resonance sweep physics when the all the lepton number is destroyed and the number of neutrinos of all kinds is fixed. We expect the latter condition to be a good approximation post Weak Decoupling. The former assumption is a good one over most of the range of neutrino masses/mixing considered here because neutrino flavor evolution is highly adiabatic for these parameters as shown above.

However, the actual energy spectra of the sterile neutrinos may come into play as precision in observations of the matter fluctuation power spectrum increase. Current constraints are most strongly dependent on the suppression of small scale power. Future constraints may be able to constrain the collisionless damping scale of neutrinosat large scales - much more strongly, and are most important in regard to spectral constraints. The collisionless damping scale for neutrinos is essentially their free streaming length, which of course depends on the neutrino velocities which, in turn, depend on the neutrino energy spectra.

\section{NON-THERMAL NEUTRINO ENERGY SPECTRA: ALTERATION OF THE RELATIONSHIP BETWEEN LEPTON NUMBERS AND PRIMORDIAL ${ }^{4} \mathrm{HE}$ ABUNDANCE}

Primordial nucleosynthesis is a freeze out from $\mathrm{Nu}-$ clear Statistical Equilibrium (NSE). In NSE the abundance of nuclei is set by a competition between disorder (entropy) and binding energy. In the early universe alpha particles win this competition because the entropy per baryon is very high and alpha particles have a binding per nucleon not terribly different from iron. At a temperature $T_{\alpha} \sim 100 \mathrm{keV}$, alpha particles form aggressively, incorporating essentially all neutrons (all but $\sim 1 / 10^{5}$ ). Therefore, the primordial ${ }^{4} \mathrm{He}$ yield is determined roughly by the neutron-to-proton ratio $n / p$ at $T_{\alpha}$. In mass fraction, this is $X_{\alpha} \approx 2(n / p) /(n / p+1)$, or $25 \%$ for $n / p=1 / 7$. The standard BBN ${ }^{4} \mathrm{He}$ mass fraction yield prediction is $(24.85 \pm 0.05) \%$ using the CMB (Cosmic Microwave Background) anisotropy-determined baryon density [6]. (The baryon closure fraction as derived from the deuterium abundance [30] is consistent with the CMB-derived value.)

The observationally inferred primordial helium abundance has a long and troubled history. One group pegs this abundance at $0.238 \pm 0.002 \pm 0.005$ [31], while another using similar but not identical compact blue galaxy data estimates $0.2421 \pm 0.0021$ [32]. These values are quite restrictive. However, these older estimates may now be superseded by more recent analyses as discussed in the Introduction. In particular, a more detailed analysis of the helium and hydrogen emission lines done in Ref. 7] suggests that the allowable range of mass fraction for primordial ${ }^{4} \mathrm{He}$ is 0.232 to 0.258 . This is fairly generous 
compared to previous "limits," but it is still a good bet that a $5 \%$ or $10 \%$ increase in the calculated, predicted yield in ${ }^{4} \mathrm{He}$ would be an unwelcome development.

The relationship between neutrino physics and/or lepton numbers and the primordial helium abundance remains a cornerstone of modern cosmology. Distortions in the $\nu_{e}$ and $\bar{\nu}_{e}$ energy spectra stemming from activesterile conversion can alter lepton capture rates on nucleons and thereby change $n / p$ and the ${ }^{4} \mathrm{He}$ yield compared to a standard BBN scenario with thermal neutrino energy spectra.

The neutron-to-proton ratio is set by the competition of the expansion rate of the universe and the rates of the following lepton capture/decay processes:

$$
\begin{aligned}
& \nu_{e}+n \rightleftharpoons p+e^{-}, \\
& \bar{\nu}_{e}+p \rightleftharpoons n+e^{+}, \\
& n \rightleftharpoons p+e^{-}+\bar{\nu}_{e} .
\end{aligned}
$$

We denote the forward and reverse rates of the first process as $\lambda_{\nu_{e} n}$ and $\lambda_{e^{-} p}$, respectively. Likewise, the forward and reverse rates of the second process are $\lambda_{\bar{\nu}_{e} p}$ and $\lambda_{e^{+} n}$, respectively, while those of the third process are $\lambda_{n}$ decay and $\lambda_{p e \bar{\nu}_{e}}$, respectively.

The lepton capture processes' influence on the isospin state of nucleons can be appreciable, even for the post Weak Decoupling epoch. This is because the number densities of relativistic neutrinos and charged leptons are some 10 orders of magnitude larger than the baryon density. At high enough temperature $(T \gg 1 \mathrm{MeV})$, where these rates are very fast, the isospin of any nucleon will flip from neutron to proton and back at a rate which is rapid compared to the expansion rate of the universe, establishing a steady state equilibrium.

As the universe expands and the temperature drops the rates of the lepton capture processes will drop off quickly. Eventually the lepton capture rates will fall below the expansion rate and $n / p$ will be frozen in, save for free neutron decay. Traditionally, this "weak freeze-out" epoch is taken to be $T_{\text {wfo }} \approx 0.7 \mathrm{MeV}$.

However, there is no sharp freeze-out of isospin. In fact, the neutron-to-proton ratio $n / p$ is modified by the lepton capture reactions down to temperatures of several hundred $\mathrm{keV}$ and by neutron decay through the epoch of alpha particle formation $T_{\alpha}$.

The evolution of the electron fraction $Y_{e}=1 /(1+n / p)$ throughout the expansion is governed by

$$
\frac{d Y_{e}}{d t}=\Lambda_{n}-Y_{e} \Lambda_{\mathrm{tot}},
$$

where the sum of the rates of the neutron destroying processes is $\Lambda_{n} \equiv \lambda_{\nu_{e} n}+\lambda_{e^{+} n}+\lambda_{n \text { decay }}$ and the sum of all weak isospin changing rates is $\Lambda_{\text {tot }} \equiv \Lambda_{n}+\lambda_{\bar{\nu}_{e} p}+$ $\lambda_{e^{-} p}+\lambda_{p e \bar{\nu}_{e}}$.

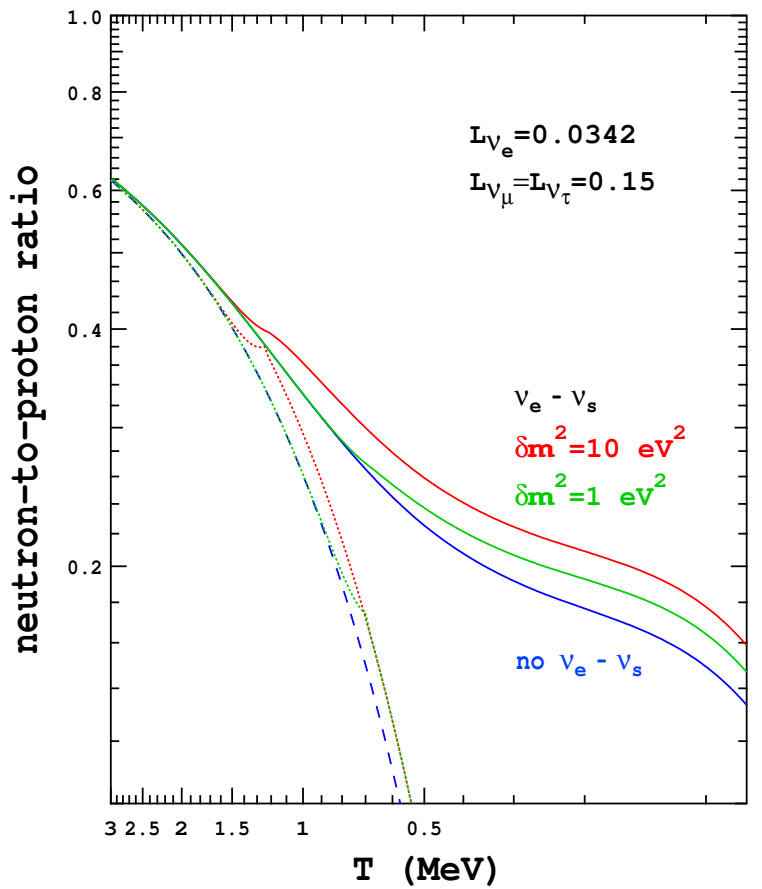

FIG. 7: The neutron to proton ratio as a function of temperature for the indicated lepton numbers. The lower solid curve gives the case with lepton numbers alone with no activesterile neutrino conversion. The dashed line is the equilibrium prediction for the $n / p$ ratio in this case. The upper solid curve is the $n / p$ ratio for the same lepton numbers, but now with active-sterile conversion in the $\nu_{e} \rightarrow \nu_{s}$ channel with $\delta m^{2}=10 \mathrm{eV}^{2}$, while the middle solid curve is for $\delta m^{2}=1 \mathrm{eV}^{2}$. The upper dotted curve is the steady state equilibrium prediction for the $n / p$ ratio for the $\delta m^{2}=10 \mathrm{eV}^{2}$ case, while the lower dotted curve gives the same prediction for the $\delta m^{2}=1 \mathrm{eV}^{2}$ case.

In the limit where the isospin flip rate is fast compared to the expansion rate $H$, the neutron-to-proton ratio has a steady state equilibrium value given by 33 ]

$$
\begin{aligned}
\frac{n}{p} & =\frac{\lambda_{\bar{\nu}_{e} p}+\lambda_{e^{-} p}+\lambda_{p e \bar{\nu}_{e}}}{\lambda_{\nu_{e} n}+\lambda_{e^{+} n}+\lambda_{n \text { decay }}}, \\
& \approx \frac{\lambda_{\bar{\nu}_{e} p}+\lambda_{e^{-} p}}{\lambda_{\nu_{e} n}+\lambda_{e^{+} n}}
\end{aligned}
$$

This solution corresponds to the fixed point $d Y_{e} / d t=0$ in Eq. (56), where $Y_{e}=\Lambda_{n} / \Lambda_{\text {tot }}$. The second approximation in Eq. (57) is valid at temperatures high enough that the rates of the three body processes can be neglected relative to the lepton capture rates.

As the universe expands and the temperature drops, the relative values of these rates change and, hence, so does the neutron-to-proton ratio. Certainly at temperatures $T>T_{\mathrm{wfo}}$ the three body lepton capture and free neutron decay processes have rates which are unimportant compared to those of the lepton capture rates. For temperatures $T \gg T_{\text {wfo }}$, typical lepton energies are large compared to the energy thresholds in the forward rate 
of the process in Eq. (54) and the reverse rate for the process in Eq. (53), so that if the lepton numbers are small we would have $n / p \approx 1$, or $Y_{e} \approx 1 / 2$. During the epoch $T_{\text {wfo }}>T>T_{\alpha}$ the lepton capture processes gradually give way to free neutron decay as the principal $n / p$-altering mechanism. Over this time period $e^{ \pm}$ annihilation heats the photon/electron plasma relative to the neutrinos, further altering the weak rates (including the free neutron decay) by modifying the neutrino and antineutrino distribution functions relative to those for $e^{ \pm}$. (See Ref. [34] for a discussion of primordial nucleosynthesis.)

If the electron neutrinos and antineutrinos and the electrons and positrons all have Fermi-Dirac energy spectra, then Eq. (57) can be reduced to 35, 36

$$
\frac{n}{p} \approx \frac{\left(\lambda_{e^{-} p} / \lambda_{e^{+} n}\right)+e^{-\eta_{\nu_{e}}+\eta_{e}-\xi}}{\left(\lambda_{e^{-} p} / \lambda_{e^{+} n}\right) e^{\eta_{\nu_{e}}-\eta_{e}+\xi}+1}
$$

where $\eta_{e}=\mu_{e} / T$ is the electron degeneracy parameter and $\xi=\left(m_{n}-m_{p}\right) / T \equiv \delta m_{n p} / T \approx 1.293 \mathrm{MeV} / T$ is the neutron-proton mass difference divided by temperature. Eq. (58) is approximate because it assumes identical neutrino and plasma temperatures. We have also neglected the neutron decay/three-body capture processes of Eq. (55). The expression in Eq. (58) is generally true for Fermi-Dirac leptonic energy distribution functions, even if the neutrinos and electrons/positrons are not in true thermal and chemical equilibrium. If and only if chemical equilibrium actually obtains (or did obtain at some early epoch) are we guaranteed to have $\mu_{e}-\mu_{\nu_{e}}=\mu_{n}-\mu_{p}$, where $\mu_{n}$ and $\mu_{p}$ are the neutron and proton total chemical potentials, respectively, and only in this case does Eq. (158) reduce to

$$
\frac{n}{p} \approx e^{\left(\mu_{e}-\mu_{\nu_{e}}-\delta m_{n p}\right) / T}
$$

With strict chemical equilibrium and with Fermi-Dirac energy distributions for all leptons, we could conclude from Eq. (59), for example, that a positive chemical potential for electron neutrinos (i.e., an excess of $\nu_{e}$ over $\bar{\nu}_{e}$ ) would suppress the steady state equilibrium neutronto-proton ratio relative to that for $\eta_{\nu_{e}}=0$. This behaviour follows also from a straightforward application of Le Chatlier's principle to the processes in Eqs. (53) and (54). A decrease in the neutron abundance translates, in turn, into a decrease in the predicted ${ }^{4} \mathrm{He}$ yield.

However, if the neutrino distribution functions are modified by active-sterile neutrino conversion, $\nu_{\alpha} \rightleftharpoons \nu_{s}$, then the resulting active and sterile neutrino distribution functions would not be Fermi-Dirac in character and we could not employ Eq. (59) to determine the neutron-toprotion ratio in steady state equilibrium. Instead, we would be forced in this case to evaluate and follow the rates directly.

We solve Eq. (56) numerically, assuming a homogeneous and isotropic FLRW universe. In these calculations we take the co-moving entropy density to be conserved and thereby calculate self consistently the $e^{ \pm}$densities and the neutrino and plasma (photon $/ e^{ \pm}$) temperatures. All neutrino energy densities are handled correctly for all assumed lepton numbers and sterile neutrino populations and, therefore, the expansion rate is also self consistently calculated. At each time step in these computations we employ appropriate neutrino, antineutrino and $e^{ \pm}$distribution functions and calculate the lepton capture rates $\lambda_{\nu_{e} n}, \lambda_{e^{-} p}, \lambda_{\bar{\nu}_{e} p}, \lambda_{e^{+} n}$, and the free neutron decay rate $\lambda_{n \text { decay. }}$. For convenience we adjust the Coulomb wave correction factor (see Appendix A) for the free neutron decay to be about $\langle G\rangle \approx 1.0227$ to give a vacuum (unblocked) neutron lifetime of $888 \mathrm{~s}$. We employ a Coulomb wave correction $\langle G\rangle=1$ for all of our lepton capture rates and we adopt an effective $f t$-value for all weak reactions $f t \approx 10^{3.035}$, so that there is roughly a $\sim 2 \%$ inconsistency in overall coupling between the lepton capture processes on the one hand and free neutron decay on the other in our calculations. This is another reason why our calculations of $n / p$ can be used only to compare trends in various cases with and without spectral distortion and not as quantitative nucleosynthesis predictions.

To gauge the effect of active-sterile neutrino flavor conversion on the evolution of the $n / p$ ratio we employ a modified forced continuous resonance sweep scenario in either the $\nu_{e} \rightarrow \nu_{s}$ or $\bar{\nu}_{e} \rightarrow \bar{\nu}_{s}$ channels. We use Eq. (25) but fix the potential lepton number at half the initial value. This will leave a low energy "hole" with zero $\nu_{e}$ (or $\bar{\nu}_{e}$ ) population, as in Fig. (1), that will grow with the expansion of the universe until $\epsilon_{\text {c.o. }}$ is reached. The final high energy edge of the "hole," $\epsilon_{\text {c.o. }}$ is determined from the initial potential lepton number as discussed in the previous sections.

The modification of the lepton capture rates in the forced continuous resonance sweep scenario is discussed in Appendix A. Consider, e.g., $\nu_{e} \rightarrow \nu_{s}$. Because in this case there are now fewer $\nu_{e}$ 's, electron capture on protons will be less Fermi blocked and, hence, the capture rate, $\lambda_{e^{-}}$, will be larger. By the same token, fewer $\nu_{e}$ 's will translate into a reduction of the $\nu_{e}$ capture rate on neutrons, $\lambda_{\nu_{e} n}$. Note that both a larger value for $\lambda_{e^{-} p}$ and a smaller value for $\lambda_{\nu_{e} n}$ go in the direction of increasing the neutron-to-proton ratio in weak steady state equilibrium. This is obvious from Eq. (57). Likewise, this trend in the rates will similarly make itself felt in the general solution of Eq. (56) and the net result will be an increase in $n / p$ at $T_{\alpha}$.

Of course, we argued above that the actual neutrino or antineutrino spectral distortions are likely to be quite different from the simple ones that continuous resonance sweep would give. Since we cannot calculate the actual neutrino spectra, we cannot give a quantitative calculation of nucleosynthesis yields. We seek here to give rough guidelines as to where one might expect significant neutrino spectral distortion modification of helium yields.

The forced continuous resonance sweep picture will serve to get the general features of the rate effects. Furthermore, it sometimes will do this in a conservative 
manner, especially for low lepton numbers where $\epsilon_{\text {c.o. }}$ is small, i.e., $\epsilon_{\text {c.o. }}<3$. As shown in Appendix A, the weak cross sections weight the square of the neutrino energy. A more realistic "picket fence" neutrino spectrum will remove population at higher neutrino energies than will the continuous sweep cut-off spectrum and will, therefore, sometimes result in larger suppression of neutrino capture rates and increases in $e^{ \pm}$capture rates. Likewise, active-active mixing will partially fill in the "hole" in the neutrino spectra, but at the cost of pushing the neutrino spectral deficit (relative to a thermal spectrum) to higher energy.

Let us consider a particular active-sterile neutrino conversion scenario in the channel $\nu_{e} \rightarrow \nu_{s}$. In this example we take each of the three neutrino flavors to have a lepton number near or at the maximum allowed without spectral distortion. We take $L_{\nu_{\mu}}=L_{\nu_{\tau}}=0.15$, corresponding to degeneracy parameters $\eta_{\nu_{\mu}}=\eta_{\nu_{\tau}} \approx 0.219$, and take $L_{\nu_{e}} \approx 0.0343$, corresponding to electron neutrino degeneracy parameter $\eta_{\nu_{e}}=0.05$. This will give an initial potential lepton number in the $\nu_{e} \rightarrow \nu_{s}$ transformation channel,

$$
\mathcal{L}_{e}^{\text {initial }}=2 L_{\nu_{e}}+L_{\nu_{\mu}}+L_{\nu_{\tau}} \approx 0.368
$$

In this case the difference in neutrino energy density over the zero lepton case is only $\sim 0.2 \%$. Therefore, the expansion rate of the universe at Weak Freeze Out in this case will differ from the standard $\mathrm{BBN}$ model by only $\sim 0.2 \%$. Therefore, the expansion rate by itself would give a negligible difference in neutron-to-proton ratio between the case with the lepton number in Eq. (60) and the zero lepton number, standard BBN case.

Nevertheless, we calculate the $n / p$ ratio completely self consistently as described above, beginning with steady state equilibrium at $T=3 \mathrm{MeV}$. The results are shown in Fig. (17). The lower solid curve is the $n / p$ ratio in the case with the lepton numbers but with no active-sterile neutrino flavor transformation and, therefore, no sterile neutrino population and no active neutrino spectral distortion. As expected, the $n / p$ ratio is suppressed relative to a zero-lepton number standard BBN calculation owing to the large positive $\nu_{e}$ degeneracy parameter $\eta_{\nu_{e}}=0.05$. At the lowest temperature on the plot, $T \approx 80 \mathrm{keV}$ (this is near $T_{\alpha}$ in an actual nucleosynthesis calculation for baryon number $\eta=6 \times 10^{-10}$ ) we have $n / p \approx 0.133$, corresponding to a rough ${ }^{4} \mathrm{He}$ yield $X_{\alpha} \approx 23.5 \%$. A similar calculation but with zero lepton numbers (this is the standard cosmological model case) gives $n / p \approx 0.141$, corresponding to $X_{\alpha}^{0} \approx 24.7 \%$, so our lepton numberonly case corresponds to about a $5 \%$ decrease in helium yield, as expected.

The dashed line on Fig. (7) is the equilibrium $n / p$ ratio given by Eq. (58) or Eq. (59) (they give the same result in this case because all leptons have Fermi-Dirac distribution functions). We see that the lepton capture rates become too slow to maintain equilibrium when $T<2 \mathrm{MeV}$, though the actual $n / p$ ratio is still significantly influenced by these rates for temperatures greater than $T \sim 300 \mathrm{keV}$.

The upper solid curve in Fig. (7) is the $n / p$ ratio in the case where the same lepton numbers now drive $\nu_{e} \rightarrow \nu_{s}$ in the modified forced continuous resonance sweep case as described above with $\delta m^{2}=10 \mathrm{eV}^{2}$. In this scenario the resonance will, eventually, sweep out to $\epsilon_{\text {c.o. }} \approx 2.724$. The upper dotted curve is the steady state equilibrium $n / p$ ratio for this case, given by Eq. (58). The "kink" in this curve (mirrored in a similar, smaller deviation in the solid curve) at about $T \approx 1.2 \mathrm{MeV}$ corresponds to the point where for this $\delta m^{2}$ and $(1 / 2)$ potential lepton number the resonance has swept far enough (i.e., near $\epsilon_{\text {c.o., }}$ see Eq. 15] to significantly reduce the $\nu_{e}+n \rightarrow$ $p+e^{-}$rate. From then on the $n / p$ ratio tracks higher than the lepton number-only case and at $T \approx 80 \mathrm{keV}$ we have $n / p \approx 0.159$, corresponding to $X_{\alpha} \approx 27.4 \%$, an $11 \%$ increase over the standard BBN zero-lepton number case, and a whopping nearly $17 \%$ increase over the lepton number-only case. (In fact, the bigger neutron number density in this case likely would lead to a slightly earlier assembly of alpha particles, i.e., a higher $T_{\alpha}$ and, hence, an even slightly bigger ${ }^{4} \mathrm{He}$ yield.)

In other words, the existence of a sterile neutrino that mixes with the $\nu_{e}$ completely altered the sign of the effect of a net lepton number. The lepton number by itself would have given a comfortable reduction of the helium yield, whereas the $\nu_{e}$ spectral distortion in this case reengineered this into an uncomfortable increase in the helium over the standard model. Despite the limitations of our calculations and approximations it is clear from this example that the existence of sterile neutrinos could alter the relationship between lepton number(s) and ${ }^{4} \mathrm{He}$ yield.

Note that there would be significant alterations in the lepton number/helium-yield relation even if the resonance did not sweep beyond $\epsilon_{\max }$. We have already argued that the resonance will find a way to sweep beyond $\epsilon_{\max }$, given that the resonance condition can be met for non-continuous resonance sweep and that neutrino flavor evolution is likely quite adiabatic for the relevant conditions. However, for argument's sake, let us adopt the same example as above and with $\delta m^{2}=10 \mathrm{eV}^{2}$, but now limit the resonance's progress to $\epsilon_{\max }$. For this example $\left(L_{\nu_{\mu}}=L_{\nu_{\tau}}=0.15\right.$ and $\left.L_{\nu_{e}} \approx 0.0343\right)$ we have $\epsilon_{\max } \approx 1.461$. The numerical $Y_{e}$ calculation in this case, using the same scenario as above, yields $n / p \approx 0.138$ at $T \approx 80 \mathrm{keV}$, a nearly $4 \%$ increase over the lepton numberonly case.

Our spectral distortion effects can be dependent on $\delta m^{2}$, at least for the high lepton numbers adopted in the above example $\left(L_{\nu_{\mu}}=L_{\nu_{\tau}}=0.15\right.$ and $\left.L_{\nu_{e}} \approx 0.0343\right)$. The middle solid curve in Fig. (7) is the $n / p$ ratio evolution for this case with forced continuous resonance sweep out to $\epsilon_{\text {c.o., }}$ but now for $\delta m^{2}=1 \mathrm{eV}^{2}$. The lower dotted curve is the Eq. (58) steady state equilibrium $n / p$ for this case. Since the resonance sweep is proportional to $\delta m^{2}$ (see Eq. 15), we expect that the resonance sweep will not have progressed far enough to decrease the $\nu_{e}$ 
capture rate significantly until a lower temperature than in the $\delta m^{2}=10 \mathrm{eV}^{2}$ case. That temperature is about $T \approx 700 \mathrm{keV}$ in this case. The result is that the rate reductions come in later here, where they are less effective at lowering $n / p$, though there is still a hefty effect. At $T \approx 80 \mathrm{keV}$ we have $n / p \approx 0.147$, corresponding to $X_{\alpha} \approx 25.6 \%$, a nearly $4 \%$ increase over our standard BBN zero-lepton number case, and a $9 \%$ increase over the lepton number-only case. If we do the same calculations but now for $\delta m^{2}=0.2 \mathrm{eV}^{2}$ we get $X_{\alpha} \approx 24.7 \%$, the same as the zero lepton number case and a $5 \%$ increase over the lepton number-only case, again a significant but smaller effect.

However, we do not see this level of $\delta m^{2}$ dependence in $n / p$ alterations when the lepton numbers are small. This is because $\epsilon_{\text {res }} \sim \delta m^{2} / \mathcal{L}$, so a low $\mathcal{L}$ translates into more progress in resonance sweep for a given $T$ and $\delta m^{2}$.

A case in point is where $L_{\nu_{e}}=L_{\nu_{\mu}}=L_{\nu_{\tau}}=0.01$ ( $\nu_{e}$ degeneracy parameter $\left.\eta_{\nu_{e}} \approx 0.0146\right)$, corresponding to potential lepton number $\mathcal{L}_{e}=0.04$ for the $\nu_{e} \rightarrow \nu_{s}$ channel, with $\epsilon_{\text {c.o. }} \approx 0.96$. This equilibrated case represents a threshold: values of $\mathcal{L}_{e}$ larger than this in fully equilibrated limit could produce significant $(>1 \%)$ increases in helium yield over the lepton number-only scenario, depending on the resonance sweep scenario and the efficacy of active-active transformation and residual neutrino down-scattering. The lepton number-only calculation for this case (as in Fig. 17) at $T \approx 79 \mathrm{keV}$ gives $n / p \approx 0.139$, or $X_{\alpha} \approx 24.4 \%$, a very slight decrease from the standard zero lepton number case discussed above. However, with $\delta m^{2} \geq 0.2 \mathrm{eV}^{2}$ and $\nu_{e} \rightleftharpoons \nu_{s}$ conversion enabled, at $T \approx 79 \mathrm{keV}$ we get $n / p \approx 0.14$, or $X_{\alpha} \approx 24.6$, a $\sim 1 \%$ increase over the lepton number-only case. Larger values of $\mathcal{L}_{e}$ will give bigger discrepancies between the cases with and without sterile neutrino mixing in the forced continuous sweep limit, and may well do so in more realistic resonance sweep scenarios as well.

Likewise, we can investigate the analogous limit for unequilibrated cases (where active-active mixing is ineffective) by employing $n / p$ evolution calculations along the lines of those presented in Fig. (7). We find that the case with $L_{\nu_{e}}=0.001$ and $L_{\nu_{\mu}}=L_{\nu_{\tau}}=0.01$ (corresponding to $\mathcal{L}_{e}=0.022, \epsilon_{\text {c.o. }} \approx 0.76$ and $\left.\eta_{\nu_{e}} \approx 0.00146\right)$ is likely to give about a $1 \%$ increase in $X_{\alpha}$ over the lepton numberonly case in the forced continuous resonance sweep scenario. Again, depending on the actual resonance sweep history and the efficacy of neutrino scattering this represents a warning point for the un-equilibrated cases.

With these numerical calculations we are led to ask the following question: at what point are the positive lepton numbers big enough that when combined with a sterile neutrino and concomitant spectral distortions we exceed the classic observationally inferred helium limits discussed above $\left(X_{\alpha}^{\lim } \approx 25 \%\right)$ ? In the forced continuous resonance sweep scenario, and taking active-active mixing as efficient, we find that limit occurs for equilibrated lepton numbers near $L_{\nu_{e}}=L_{\nu_{\mu}}=L_{\nu_{\tau}}=0.09\left(\nu_{e}\right.$ degeneracy parameter $\eta_{\nu_{e}} \approx 0.131$ ), corresponding to po-

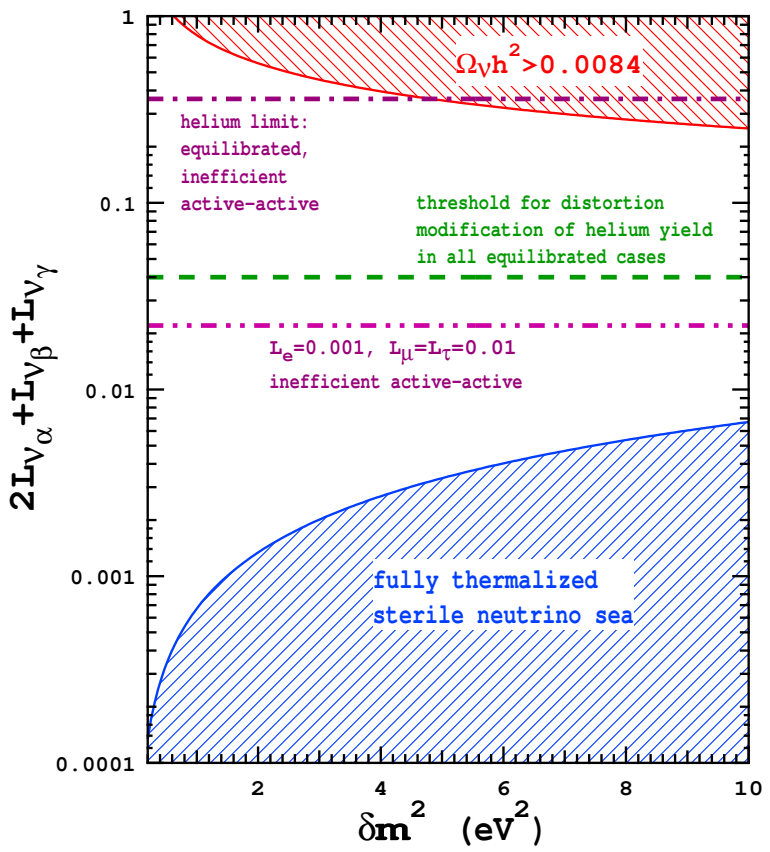

FIG. 8: Constraints as in Fig. (6) and now with BBN "guidelines," see text. The double-dot-dashed line gives an estimate of the limiting ${ }^{4} \mathrm{He}$ yield in a no active-active mixing scenario where $L_{e}=0.001$ and $L_{\mu}=L_{\tau}=0.01$. The dashed line gives a threshold in potential lepton number for all cases where neutrino lepton numbers are equilibrated, including those with efficient active-active mixing. Beyond this threshold there could be significant alterations in the helium yield stemming from non-thermal neutrino distributions. The upper dash-dot line gives a rough estimate of the limiting potential lepton number for equilibrated cases with inefficient active-active mixing.

tential lepton number $\mathcal{L}_{e}=0.36$ for the $\nu_{e} \rightarrow \nu_{s}$ channel, with $\epsilon_{\text {c.o. }} \approx 2.57$. The lepton number-only calculation for these parameters gives $n / p \approx 0.121$ or $X_{\alpha} \approx 21.6 \%$ at $T \approx 79 \mathrm{keV}$; whereas, active-sterile transformation in this case with $\delta m^{2}=10 \mathrm{eV}^{2}$ yields at this temperature $n / p \approx 0.143$ or $X_{\alpha} \approx 25 \%$. However, this drops to $X_{\alpha} \approx 24.6 \%$ for $\delta m^{2}=5 \mathrm{eV}^{2}$ and is down to $X_{\alpha} \approx 23 \%$ for $\delta m^{2}=0.2 \mathrm{eV}^{2}$. For these lepton number parameters we note that $\delta m^{2}>5 \mathrm{eV}^{2}$ may already be ruled out by closure constraints.

These rough cautionary guides as to where one might expect sterile neutrino-generated spectral distortions to become important are shown superposed on the closure fraction constraints in Fig. (8). Our calculations are only very general guides, as discussed above, as we cannot follow the resonance sweep physics in detail and, therefore, we cannot be quantitative about nucleosynthesis yields.

One example of a significant deviation from the forced resonance sweep picture is where active-active neutrino mixing is efficient and neutrino lepton numbers equilibrate rapidly as resonance sweep occurs. In particular, in Cases 1 and 2 discussed in the last section, the neutrino populations in the "hole" are not zero, with the conse- 
quence that for a given $\epsilon_{\text {c.o. }}$ the rates are not as affected as in the cases discussed above. For example, for initial lepton numbers $L_{\nu_{e}}=L_{\nu_{\mu}}=L_{\nu_{\tau}}=0.1$, corresponding to $\eta_{\nu_{e}} \approx 0.146$, the lepton number-only case steady state $n / p$ ratio from Eq. (59) at $T=0.7 \mathrm{MeV}$ is $n / p \approx 0.136$. By contrast, when we allow sterile neutrino production in Case 1, the resulting non-thermal $\nu_{e}$ spectrum increases the steady state value to $n / p \approx 0.15$. In Case 2 this goes to $n / p \approx 0.145$ while in Case 3 it is only $n / p \approx 0.144$. These examples serve to illustrate the change in lepton capture rates between Cases 1, 2, and 3. This gives a rough guide as to how spectral distortion effects on BBN might decrease in calculations with the full $Y_{e}$ evolution as we go from Case 1 toward Case 3 .

Likewise, the cases with the highest values of $\delta m^{2}$ coupled with lower values of $\mathcal{L}$ in Fig. (8) may already have experienced considerable resonant active-sterile conversion and concomitant lepton number depletion before Weak Decoupling, while active neutrino scattering downscattering was still effective. This would either wash out much of the "hole" in the neutrino distribution functions or leave a hole of reduced energy width at higher energies.

What about conversion of $\bar{\nu}_{e}$ to steriles, i.e., $\bar{\nu}_{e} \rightarrow \bar{\nu}_{s}$ ? This process can be matter-enhanced when the overall potential lepton number is negative, $\mathcal{L}_{e}<0$. It will be exactly analogous to the positive potential lepton number case, at least as far as the neutrino flavor conversion and the resonance sweep physics goes. This close analogy ends, however, when it comes to the lepton capture reactions.

If the resonance sweeps adiabatically in the forced resonance sweep scenario out to a scaled antineutrino energy $\bar{\epsilon}_{\text {c.o. }}$, a hole will be left in the $\bar{\nu}_{e}$ distribution, in complete analogy to the cases discussed above. Fewer $\bar{\nu}_{e}$ 's will translate into a decreased antineutrino capture rate, $\bar{\nu}_{e}+p \rightarrow n+e^{+}$, and an increased rate of positron capture, $e^{+}+n \rightarrow p+\bar{\nu}_{e}$. These rate modifications both go in the direction of decreasing the neutron-to-proton ratio $n / p$, as is obvious from Eq. (57).

One might think at first that simply changing the sign of the potential lepton numbers given in the above examples could result in a significantly suppressed $n / p$ at $T_{\alpha}$. This is not necessarily correct however, because the threshold in the reaction $\bar{\nu}_{e}+p \rightarrow n+e^{+}$plays a crucial role. As can be seen in the rate integrals given in Appendix $\mathrm{A}$, in this channel a $\bar{\nu}_{e}$ must have an energy in excess of the threshold, $E_{\bar{\nu}_{e}}>E_{\bar{\nu}_{e}}^{\text {thresh }}$ to be captured. The threshold is $E_{\bar{\nu}_{e}}^{\text {thresh }}=Q_{n p}+m_{e} c^{2} \approx 1.804 \mathrm{MeV}$.

In fact, in the forced continuous resonance sweep scenario, unless $\bar{\epsilon}_{\text {c.o. }}>E_{\bar{\nu}_{e}}^{\text {thresh }} / T$, there will be no modifications in the $\bar{\nu}_{e}$ capture rates. Likewise for the inverse process of positron capture on neutrons, $e^{+}+n \rightarrow p+\bar{\nu}_{e}$. In this reaction, there will be no alteration of the final state $\bar{\nu}_{e}$ blocking factor unless $\bar{\epsilon}_{\text {c.o. }}>E_{\bar{\nu}_{e}}^{\text {thresh }} / T$.

For example, consider the equilibrated case with $L_{\nu_{e}}=$ $L_{\nu_{\mu}}=L_{\nu_{\tau}}=-0.01\left(\eta_{\nu_{e}} \approx-0.0146\right)$. This gives the opposite sign potential lepton number, $\mathcal{L}_{e} \approx-0.04$, from the analogous positive lepton number case considered

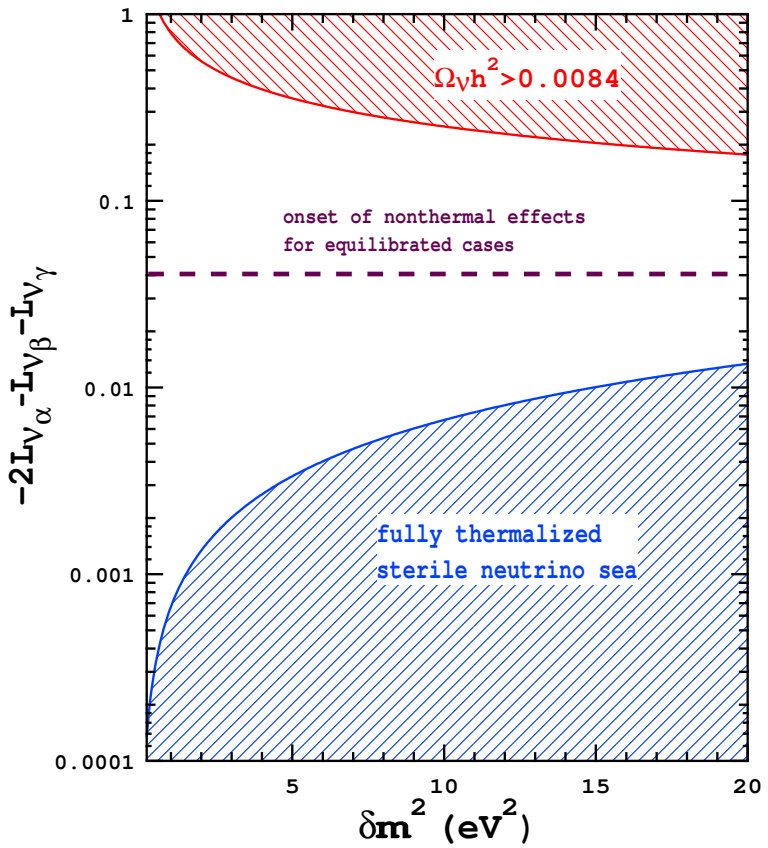

FIG. 9: Constraints and guidelines as in Fig.s (6) \& (8), but now for negative values of potential lepton number. The dashed line gives a rough threshold on potential lepton number in the equilibrated regime $\left(L_{e}=L_{\mu}=L_{\tau}\right)$ beyond which significant alterations (reductions) in ${ }^{4} \mathrm{He}$ yield stemming from $\bar{\nu}_{e}$ spectral distortion might be expected.

above. The forced continuous, adiabatic resonance sweep scenario would give for this case $\bar{\epsilon}_{\text {c.o. }} \approx 0.96$. A calculation of $Y_{e}$ with temperature as in Fig. (7) for the lepton number-only version of this case gives $n / p \approx 0.148$ at $T \approx 79 \mathrm{keV}$, or roughly $X_{\alpha} \approx 25.7 \%$, an increase in helium yield over the standard zero lepton number case, as expected. However, when we now do the same calculation but with $\bar{\nu}_{e} \rightarrow \bar{\nu}_{s}$ conversion with $\delta m^{2}>0.2 \mathrm{eV}^{2}$ we obtain at the same temperature $n / p \approx 0.143$, corresponding to $X_{\alpha} \approx 25 \%$. This is a $\sim 3 \%$ reduction over the lepton number-only case.

This negative potential lepton number value again signals a threshold: negative potential lepton numbers in the fully equilibrated limit larger in magnitude than this could give significant modification of the relationship between lepton number and helium yield. This guideline is shown in Fig. (9) in the same manner as for the guides for positive potential lepton numbers. Again, the warning as to the very rough nature of our guidelines owing to uncertain resonance sweep physics and the efficacy of neutrino down-scattering and active-active mixing applies here as well as in Fig. (8).

Because of the threshold issue in the negative potential lepton number cases it is not possible to find parameters that could provide a large suppression of ${ }^{4} \mathrm{He}$ over the zero lepton number case, at least in the fully equilibrated limit. Partly this is because large lepton number magnitude would require a large $\delta m^{2}$ in order to have enough 
resonance sweep early enough to affect the lepton capture enough to drop $n / p$ significantly. The large $\delta m^{2}$ at these large, negative $\mathcal{L}_{e}$ values seem to be in conflict with closure constraints.

\section{DISCUSSION AND CONCLUSIONS}

The most general conclusions that can be drawn from this work is that the existence of one or more light sterile neutrinos could (1) alter the relationship between neutrino chemical potential and primordial nucleosynthesis yields, and (2) leave both active and sterile neutrinos with nonthermal, distorted energy spectra. Attempts to constrain an "LSND" sterile neutrino based on conventional degenerate primordial nucleosynthesis considerations, as well as attempts to reconcile this neutrino with BBN limits via a primordial lepton number are now suspect. However, obtaining the detailed relationship between lepton numbers, active-sterile neutrino mixing parameters and light element nucleosynthesis yields that would be required to effect reliable constraints is beyond the scope of the work presented in this paper.

This is mostly a consequence of another discovery made in this work: MSW resonances cannot sweep smoothly and continuously beyond $\epsilon_{\max }$. We showed that one way the resonance condition and adiabatic conversion criterion can be met beyond this point is if the resonance skips to higher energies a number of times until the initial lepton number is depleted. Partly for this reason resonance evolution is likely to be quite complicated. Complication will also arise because active-active neutrino mixing can be efficient and can occur simultaneously with active-sterile transformation.
In any case, neutrino flavor conversion is almost inevitable once the resonance condition is met. This is on account of another insight presented in this paper: the highly adiabatic nature of neutrino flavor evolution through MSW resonances for the neutrino mass/mixing parameters of most interest and for the conditions in the post Weak Decoupling early universe.

If the mini-BooNE experiment sees a positive signal, confirming the existence of light sterile neutrinos, we will be forced to confront the problems posed in this paper. Likewise, future progress in improving the precision and confidence in the observationally inferred primordial helium abundance coupled with $\mathrm{CMB}$ and large scale structure-derived limits on neutrino collisionless damping scales could give us hints about active-sterile neutrino mixing physics in the early universe.

\section{Acknowledgments}

The work of G.M.F. was supported in part by NSF grant PHY-00-99499, the TSI collaboration's DOE SciDAC grant, and a UC/LANL CARE grant at UCSD; K.A. was supported by Los Alamos National Laboratory (under DOE contract W-7405-ENG-36) and also acknowledges the UC/LANL CARE grant. N.B. was supported by Fermilab, which is operated by URA under DOE contract No. DE-AC02-76CH03000, and was additionally supported by NASA under NAG5-10842. This research was also supported in part by the NSF under Grant No. PHY99-07949. The authors thank the ECT* and the INT at the University of Washington for hospitality. We thank Ray Volkas and Robert Foot for discussions.
[1] K. Eitel, New J. Phys. 2, 1 (2000).

[2] G. McGregor [MiniBooNE Collaboration], AIP Conf. Proc. 655, 58 (2003).

[3] H. Murayama and T. Yanagida, Phys. Lett. B 520, 263 (2001); G. Barenboim, L. Borissov, J. Lykken and A. Y. Smirnov, JHEP 0210, 001 (2002).

[4] M. C. Gonzalez-Garcia, M. Maltoni and T. Schwetz, Phys. Rev. D 68, 053007 (2003).

[5] K. N. Abazajian, Astropart. Phys. 19, 303 (2003); P. Di Bari, Phys. Rev. D 65, 043509 (2002) [Addendum-ibid. D 67, 127301 (2003)].

[6] R. H. Cyburt, B. D. Fields, K. A. Olive and E. Skillman, Astropart. Phys. 23, 313 (2005).

[7] K. A. Olive and E. D. Skillman, Astrophys. J. 617, 29 (2004).

[8] S. Hannestad, JCAP 0305, 004 (2003); . Pierce and H. Murayama, Phys. Lett. B 581, 218 (2004).

[9] M. Tegmark et al. [SDSS Collaboration], Phys. Rev. D 69, 103501 (2004). D. N. Spergel et al., Astrophys. J. Suppl. 148, 175 (2003).

[10] U. Seljak et al., Phys. Rev. D 71, 103515 (2005).

[11] G. M. Fuller and D. N. Schramm, Phys. Rev. D 45, 2595
(1992).

[12] Z. Chacko, L. J. Hall, S. J. Oliver and M. Perelstein, Phys. Rev. Lett. 94, 111801 (2005).

[13] Z. Chacko, L. J. Hall, T. Okui and S. J. Oliver, Phys. Rev. D 70, 085008 (2004); R. N. Mohapatra and S. Nasri, Phys. Rev. D 71, 053001 (2005).

[14] J. F. Beacom, N. F. Bell and S. Dodelson, Phys. Rev. Lett. 93, 121302 (2004).

[15] R. Foot and R. R. Volkas, Phys. Rev. Lett. 75, 4350 (1995).

[16] S.P. Mikheyev and A.Yu. Smirnov, Yad. Fiz. 42, 1441 (1985) [Sov. J. Nucl. Phys. 42, 913 (1985)]; L. Wolfenstein, Phys. Rev. D 17, 2369 (1978).

[17] A. D. Dolgov, S. H. Hansen, S. Pastor, S. T. Petcov, G. G. Raffelt and D. V. Semikoz, Nucl. Phys. B 632, 363 (2002); K. N. Abazajian, J. F. Beacom and N. F. Bell, Phys. Rev. D 66, 013008 (2002); Y. Y. Y. Wong, Phys. Rev. D 66, 025015 (2002).

[18] V. Barger, J. P. Kneller, P. Langacker, D. Marfatia and G. Steigman, Phys. Lett. B 569, 123 (2003);

[19] J. P. Kneller, R. J. Scherrer, G. Steigman and T. P. Walker, Phys. Rev. D 64, 123506 (2001). 
[20] D. P. Kirilova, Astropart. Phys. 19, 409 (2003).

[21] K. Abazajian, X. Shi and G. M. Fuller, arXiv:astro-ph/9909320

[22] M.Yu.Khlopov and S.T.Petcov Phys. Lett., B99, 117 (1981); Ya.B.Zeldovich and M.Yu.Khlopov Usp. Fiz. Nauk 135, 45 (1981). [English translation: Sov. Phys. Uspekhi 24, 755 (1981).]

[23] M. Cirelli, G. Marandella, A. Strumia and F. Vissani, Nucl. Phys. B 708, 215 (2005) arXiv:hep-ph/0403158.

[24] D. Notzold and G. Raffelt, Nucl. Phys. B 307, 924 (1988).

[25] X. Shi and G. M. Fuller, Phys. Rev. Lett. 82, 2832 (1999).

[26] Y.-Z. Qian and G. M. Fuller, Phys. Rev. D 51, 1479 (1995).

[27] A. B. Balantekin and G. M. Fuller, Phys. Lett. B471, 195 (1999).

[28] G. C. McLaughlin, J. M. Fetter, A. B. Balantekin and G. M. Fuller, Phys. Rev. C 59, 2873 (1999).

[29] M. Sorel, J. M. Conrad and M. Shaevitz, Phys. Rev. D 70, 073004 (2004).

[30] J. M. O'Meara, D. Tytler, D. Kirkman, N. Suzuki, J. X. Prochaska, D. Lubin and A. M. Wolfe, Astrophys. J. 552, 718 (2001).

[31] K. A. Olive, E. Skillman and G. Steigman, Astrophys. J. 483, 788 (1997).

[32] Y. I. Izotov and T. X. Thuan, Astrophys. J. 602, 200 (2004).

[33] Y. Z. Qian, G. M. Fuller, G. J. Mathews, R. Mayle, J. R. Wilson and S. E. Woosley, Phys. Rev. Lett. 71, 1965 (1993).

[34] M. S. Smith, L. H. Kawano and R. A. Malaney, Astrophys. J. Suppl. 85, 219 (1993).

[35] C. Y. Cardall and G. M. Fuller, Astrophys. J. 472, 435 (1996).

[36] G. M. Fuller, W. A. Fowler, M. J. Newman, Astrophys. J. 293, 1 (1985).

\section{APPENDIX A: WEAK RATES WITH NON-THERMAL NEUTRINO ENERGY SPECTRA}

In this Appendix we calculate the forward and reverse rates of the processes in Eqs. (53) \& (54) for the cases where, respectively, all neutrinos below energy $E_{\nu_{e}}=T \epsilon$ or antineutrinos below energy $E_{\bar{\nu}_{e}}=T \bar{\epsilon}$ are converted to sterile species. We provide estimates of these rates in terms of standard relativistic Fermi integrals. We also discuss how these rates would be modified if the MSW resonance does not sweep smoothly and continuously (and adiabatically) through the low energy neutrino or antineutrino distribution function, but instead skips to higher energies. The rate modifications for Cases 1 and 2 in the efficient active-active neutrino mixing limit will be different, of course, because in those scenarios the "holes" in the neutrino distribution functions are not empty. Though the rate formulae presented here are not valid for these cases, they still give a general idea of how the lepton capture/decay rates depend on spectral distortion and thresholds.

If there is no active-sterile conversion and all neutrino, nucleon, and charged lepton distribution functions are thermal in character, the $\nu_{e}$ capture rate on neutrons is $\lambda_{\nu_{e} n}^{0}$. By contrast, we will denote as $\lambda_{\nu_{e} n}$ the actual electron neutrino capture rate when the same thermodynamic conditions obtain, but now where $\nu_{e}$ 's have been converted to sterile species up to scaled energy $\epsilon$ as outlined above. If all neutrino, nucleon, and charged lepton energy distribution functions are at least piece-wise Fermi-Dirac or zero, these rates can be written [36], respectively, as

$$
\begin{aligned}
\lambda_{\nu_{e} n}^{0} & \approx \Lambda\left[1-e^{\eta_{e}-\eta_{\nu_{e}}-\xi_{n p}}\right]^{-1} \int_{0}^{\infty} x^{2}\left(x+\xi_{n p}\right)^{2}\left(\frac{1}{e^{x-\eta_{\nu_{e}}}+1}-\frac{1}{e^{x+\xi_{n p}-\eta_{e}}+1}\right) d x \\
\lambda_{\nu_{e} n} & \approx \Lambda\left[1-e^{\eta_{e}-\eta_{\nu_{e}}-\xi_{n p}}\right]^{-1} \int_{\epsilon}^{\infty} x^{2}\left(x+\xi_{n p}\right)^{2}\left(\frac{1}{e^{x-\eta_{\nu_{e}}}+1}-\frac{1}{e^{x+\xi_{n p}-\eta_{e}}+1}\right) d x \\
& \approx \Lambda\left[1-e^{\eta_{e}-\eta_{\nu_{e}}-\xi_{n p}}\right]^{-1} \sum_{n=0}^{4} \alpha_{n}\left[F_{n}\left(\eta_{\nu}^{\mathrm{eff}}\right)-F_{n}\left(\eta_{e}^{\mathrm{eff}}\right)\right] .
\end{aligned}
$$

Here the integration variable in both equations is the scaled $\nu_{e}$ energy, $x=E_{\nu_{e}} / T$. The final state electron energy is $E_{e}=T\left(x+\xi_{n p}\right)$. The other notation in these expressions is as defined above and $\xi_{n p} \equiv Q_{n p} / T$ with 
$Q_{n p}=\delta m_{n p}$. There is no threshold for $\nu_{e}$ energy in this reaction channel. The temperature and matrix elementdependent factor in both rate expressions is

$$
\begin{aligned}
\Lambda & \equiv\langle G\rangle \frac{\ln 2}{\langle f t\rangle}\left(\frac{T}{m_{e} c^{2}}\right)^{5} \\
& \approx\left(1.835 \times 10^{-2} \mathrm{~s}^{-1}\right)\langle G\rangle\left(\frac{T}{\mathrm{MeV}}\right)^{5}
\end{aligned}
$$

where $\langle f t\rangle$ is the effective $f t$-value as defined in Ref. 36 and is roughly $\log _{10} f t \approx 3.035$ for free nucleons, while $\langle G\rangle$ is the average Coulomb wave correction factor (also defined in Ref. [36]) with $G \equiv F\left(Z, E_{e}\right) E_{e} / p_{e}$ and where $F\left(Z, E_{e}\right)$ is the usual Fermi function for nuclear charge $Z$ and final state electron energy $E_{e}$. For the relativistic leptons considered here (the lowest electron energy is $\approx$ $\left.Q_{n p} \approx 1.3 \mathrm{MeV}\right),\langle G\rangle \approx 1$, though we note that $\langle G\rangle$ in the no-transformation case is slightly larger than that for the case with the $\epsilon$ cut-off on account of the lower energy electrons present in the phase space integral in the former case. (Electrons are "pulled in" to the proton because of Coulomb attraction, making for a larger overlap.)

The second approximation in Eq. A2 gives $\lambda_{\nu_{e} n}$ as a sum of differences of relativistic Fermi integrals. In this expression the effective $\nu_{e}$ and $e^{-}$degeneracy parameters are defined as $\eta_{\nu}^{\text {eff }} \equiv \eta_{\nu_{e}}-\epsilon$ and $\eta_{e}^{\text {eff }} \equiv \eta_{e}-\delta$, respectively, where $\delta \equiv \epsilon+\xi_{n p}$. Also in Eq. (A2) we define $\alpha_{4} \equiv$ 1 , while $\alpha_{3} \equiv 2(\epsilon+\delta)$, and $\alpha_{2} \equiv(\epsilon+\delta)^{2}+2 \epsilon \delta$, with $\alpha_{1} \equiv 2 \epsilon \delta(\epsilon+\delta)$ and $\alpha_{0} \equiv \epsilon^{2} \delta^{2}$. Note that as $\epsilon \rightarrow 0$, both expressions in Eq. (A2) approach $\lambda_{e^{-} p}^{0}$ in Eq. (A1). It is obvious that for nonzero $\epsilon$ the $\nu_{e}$ capture rate on neutrons will be reduced over its no-transformation value, $\lambda_{\nu_{e} n}<\lambda_{\nu_{e} n}^{0}$. The rate $\lambda_{\nu_{e} n}$ is illustrated in Fig. [10] as a function of $\epsilon$ or $L_{\nu_{e}}$.

The rate for the corresponding reverse process of electron capture on protons, $e^{-}+p \rightarrow n+\nu_{e}$, will be increased if some $\nu_{e}$ 's are transformed to sterile states, as there will be less final state $\nu_{e}$ blocking in this case. For a Fermi-Dirac distribution of electrons, and in terms of an integral over electron energy $E_{e}$, this rate is

$$
\lambda_{e^{-} p} \approx \frac{\langle G\rangle \ln 2}{\langle f t\rangle\left(m_{e} c^{2}\right)^{5}} \int_{Q_{n p}}^{\infty} \frac{E_{e}^{2}\left(E_{e}-Q_{n p}\right)^{2}}{e^{E_{e} / T-\eta_{e}}+1}\left[1-S_{\nu_{e}}\right] d E_{e}
$$

where $S_{\nu_{e}}$ is the energy-dependent $\nu_{e}$ occupation probability,

$$
\begin{array}{ll}
S_{\nu_{e}}=0 & \text { for } E_{\nu_{e}} / T \leq \epsilon, \\
S_{\nu_{e}}=\frac{1}{e^{E_{\nu_{e}} / T-\eta_{\nu_{e}}}+1} & \text { for } E_{\nu_{e}} / T>\epsilon .
\end{array}
$$

Here the $\nu_{e}$ energy is $E_{\nu_{e}}=E_{e}-Q_{n p}$ on account of the threshold, $Q_{n p}$.

It is convenient to re-write the rate in Eq. A4 as an integration over neutrino energy scaled by temperature, $x=E_{\nu_{e}} / T$, and as a sum of contributions from low neutrino energy with no final state blocking, and higher

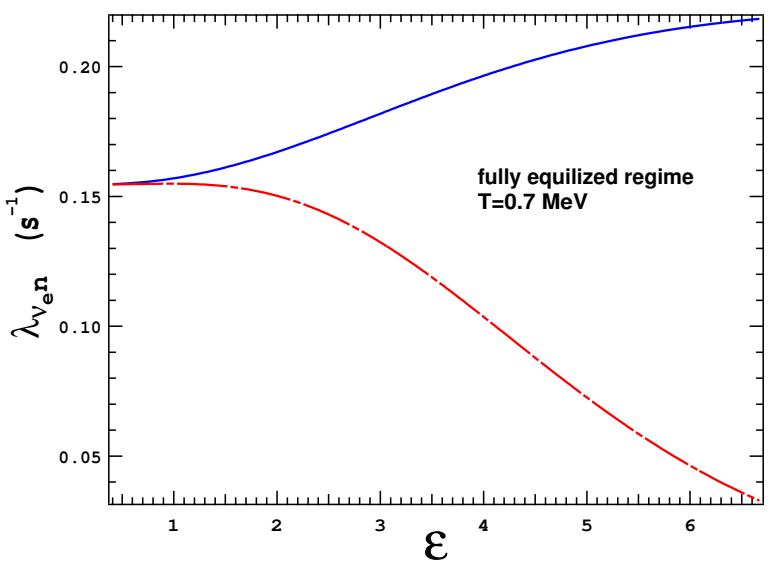

FIG. 10: Rate $\lambda_{\nu_{e} n}$ in $\mathrm{s}^{-1}$ for the process $\nu_{e}+n \rightarrow p+e^{-}$at temperature $T=0.7 \mathrm{MeV}$ as a function of $\epsilon$ and/or $L_{\nu_{e}}$ in the smooth and continuous resonance sweep limit and for the case of complete active neutrino equalization $\left(L_{\nu_{e}}=L_{\nu_{\mu}}=L_{\nu_{\tau}}\right)$. The solid curve gives the rate for no sterile neutrino conversion, thermal $\nu_{e}$ distribution, but with the $\nu_{e}$ chemical potential appropriate for the corresponding $\epsilon$ value. The dotdashed curve gives the rate with active-sterile neutrino conversion and corresponding non-thermal character for the $\nu_{e}$ energy distribution function.

final state neutrino energy where there is non-zero Fermi blocking,

$$
\lambda_{e^{-} p}=\lambda_{e^{-} p}^{\mathrm{low}}+\lambda_{e^{-} p}^{\mathrm{high}}
$$

The first of these rate contributions can be approximated by

$$
\lambda_{e^{-} p}^{\text {low }} \approx \Lambda \int_{0}^{\epsilon} \frac{x^{2}\left(x+\xi_{n p}\right)^{2}}{e^{x+\xi_{n p}-\eta_{e}}+1} d x .
$$

Just as for $\nu_{e}$ capture, the average Coulomb wave correction factor will be lower (closer to unity) with increasing $\epsilon$. Again this has to do with the enhancement of the low energy electron probability density near the proton. As above, we can represent the rate contribution in Eq. A8 in terms of standard relativistic Fermi integrals,

$$
\lambda_{e^{-} p}^{\text {low }} \approx \Lambda\left[F_{4}\left(\eta_{e}-\xi_{n p}\right)+2 \xi_{n p} F_{3}\left(\eta_{e}-\xi_{n p}\right)+\xi_{n p}^{2} F_{2}\left(\eta_{e}-\xi_{n p}\right)\right]-\Lambda \sum_{n=0}^{4} \beta_{n} F_{n}\left(\eta_{e}-\xi_{n p}-\epsilon\right),
$$




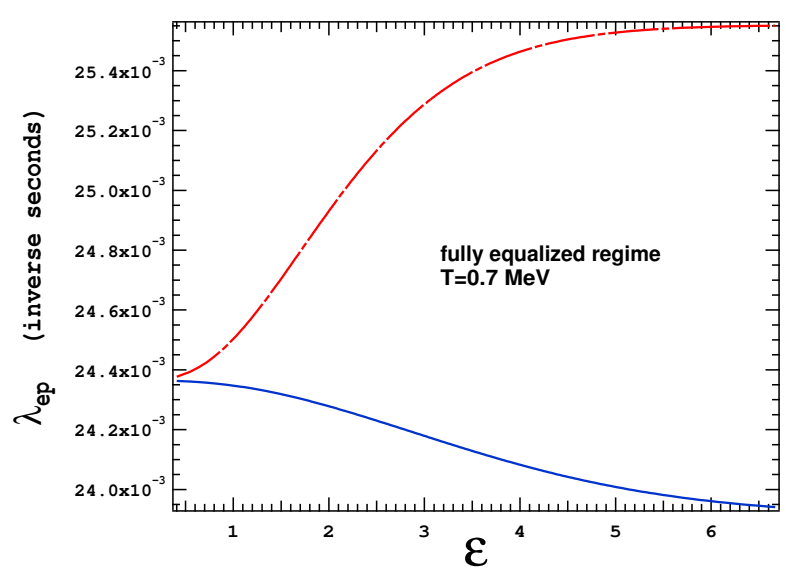

FIG. 11: Rate $\lambda_{e p}$ in $\mathrm{s}^{-1}$ for the process $e^{-}+p \rightarrow n+\nu_{e}$ at temperature $T=0.7 \mathrm{MeV}$ as a function of $\epsilon$ and/or $L_{\nu_{e}}$ in the smooth and continuous resonance sweep limit and for the case of complete active neutrino equalization $\left(L_{\nu_{e}}=L_{\nu_{\mu}}=L_{\nu_{\tau}}\right)$. The solid curve gives the rate for no sterile neutrino conversion, thermal $\nu_{e}$ distribution, but with the $\nu_{e}$ chemical potential appropriate for the corresponding $\epsilon$ value. The dotdashed curve gives the rate with active-sterile neutrino conversion and corresponding non-thermal character of the $\nu_{e}$ energy distribution function. where $\beta_{4} \equiv 1$, and where $\beta_{3} \equiv 2(\epsilon+\delta)$, while $\beta_{2} \equiv$ $(\epsilon+\delta)^{2}+2 \epsilon \delta$ and $\beta_{1} \equiv 2 \epsilon \delta(\epsilon+\delta)$, with $\beta_{0} \equiv \epsilon^{2} \delta^{2}$. Here we define $\delta \equiv \epsilon+\xi_{n p}$.
The physical interpretation of this expression for $\lambda_{e^{-} p}^{\text {low }}$ is clear if it is recalled that the $\nu_{e}$ energy is $E_{\nu_{e}}=$ $E_{e}-Q_{n p}$, implying that the "effective final state neutrino degeneracy parameter" is $\eta_{e}-\xi_{n p}$ for the no-conversion case, and $\eta_{e}-\xi_{n p}-\epsilon$ with conversion of $\nu_{e}$ 's to steriles. Of course, as $\epsilon \rightarrow 0$, the rate contribution from the (final state $\left.\nu_{e}\right)$ unblocked portion of the phase space approaches zero, $\lambda_{e^{-} p}^{\text {low }} \rightarrow 0$. The second of the rate contributions in Eq. (A7) can be approximated as

$$
\begin{aligned}
\lambda_{e^{-} p}^{\text {high }} & \approx \Lambda\left[1-e^{\xi_{n p}-\eta_{e}+\eta_{\nu_{e}}}\right]^{-1} \int_{\epsilon}^{\infty} x^{2}\left(x+\xi_{n p}\right)^{2}\left(\frac{1}{e^{x+\xi_{n p}-\eta_{e}}+1}-\frac{1}{e^{x-\eta_{\nu_{e}}}+1}\right) d x \\
& \approx \Lambda\left[1-e^{\xi_{n p}-\eta_{e}+\eta_{\nu_{e}}}\right]^{-1} \sum_{n=0}^{4} \beta_{n}\left[F_{n}\left(\eta_{e}-\xi_{n p}-\epsilon\right)-F_{n}\left(\eta_{\nu_{e}}-\epsilon\right)\right]
\end{aligned}
$$

where the notation is as above and where the $\beta_{n}$ are as defined above for Eq. A9 . In summary, a hole in the low energy $\nu_{e}$ distribution results in a lower value for $\lambda_{\nu_{e} n}$, a higher value for $\lambda_{e^{-} p}$ and, hence, an increased $n / p$ ratio. The rate $\lambda_{e^{-} p}$ is illustrated in Fig. 11] as a function of $\epsilon$ or $L_{\nu_{e}}$.

By contrast, conversion up to scaled energy $\bar{\epsilon}=E_{\bar{\nu}_{e}} / T$ of $\bar{\nu}_{e}$ 's to sterile neutrinos, $\bar{\nu}_{e} \rightarrow \bar{\nu}_{s}$, would result in a lower value of the neutron-to-proton ratio and, hence, a lower ${ }^{4} \mathrm{He}$ yield. This is because a low energy deficit in the $\bar{\nu}_{e}$ distribution would lead to a decreased rate for $\bar{\nu}_{e}+p \rightarrow n+e^{+}$and, on account of less blocking, an increased rate for the reverse process. Handling the energy threshold for these reactions is, however, somewhat more complicated than for $\nu_{e}$ and $e^{-}$capture.

Using much the same notation as above, we can approximate the rate for $\bar{\nu}_{e}+p \rightarrow n+e^{+}$as

$$
\begin{aligned}
& \lambda_{\bar{\nu}_{e} p} \approx \Lambda\left[1-e^{\xi_{n p}-\eta_{e}-\eta_{\bar{\nu}_{e}}}\right]^{-1} \int_{\gamma_{\text {thresh }}}^{\infty} x^{2}\left(x-\xi_{n p}\right)^{2}\left(\frac{1}{e^{x-\eta_{\bar{\nu}_{e}}+1}}-\frac{1}{e^{x-\xi_{n p}+\eta_{e}}+1}\right) d x \\
& \approx \Lambda\left[1-e^{\xi_{n p}-\eta_{e}-\eta_{\bar{\nu}}}\right]^{-1} \sum_{n=0}^{4} \bar{\alpha}_{n}\left[F_{n}\left(\eta_{\bar{\nu}}^{\text {eff }}\right)-F_{n}\left(\eta_{\bar{e}}^{\text {eff }}\right)\right] .
\end{aligned}
$$

The integration variable in the first of these equations is $x=E_{\bar{\nu}_{e}} / T$, and the final state positron energy will be 
$E_{e^{+}}=T\left(x-\xi_{n p}\right)$. The scaled energy threshold in these expressions is

$$
\begin{array}{ll}
\gamma_{\text {thresh }}=\xi_{n p}+m_{e} & \text { for } \xi_{n p}+m_{e} \geq \bar{\epsilon} \\
\gamma_{\text {thresh }}=\bar{\epsilon} & \text { for } \bar{\epsilon}>\xi_{n p}+m_{e}
\end{array}
$$

where $m_{e} \equiv m_{e} c^{2} / T$. It is clear that transformation of $\bar{\nu}_{e}$ 's with energies below the threshold energy $Q_{n p}+m_{e} c^{2}$ does not affect the rate. In the second approximation in Eq. A11, the effective $\bar{\nu}_{e}$ degeneracy parameter is $\eta_{\bar{\nu}}^{\text {eff }}=$ $\eta_{\bar{\nu}_{e}}-\bar{\epsilon}$, while the effective positron degeneracy parameter is $\eta_{\bar{e}}^{\mathrm{eff}}=\xi_{n p}-\eta_{e}-\bar{\epsilon}$. (Since electromagnetic equilibrium always obtains here, the positron and electron degeneracy parameters have equal magnitudes and opposite signs, $\left.\eta_{e^{+}}=-\eta_{e}.\right)$ If we define $a \equiv 2 \bar{\epsilon}-\xi_{n p}$ and $b \equiv \bar{\epsilon}\left(\bar{\epsilon}-\xi_{n p}\right)$, then the coefficients $\bar{\alpha}_{n}$ are: $\bar{\alpha}_{4}=1 ; \bar{\alpha}_{3}=2 a ; \bar{\alpha}_{2}=$ $a^{2}+2 b ; \bar{\alpha}_{1}=2 a b ;$ and $\bar{\alpha}_{0}=b^{2}$.

Utilizing the same quantities and notation as in Eq. (A11), the rate for the reverse process of positron capture, $e^{+}+n \rightarrow p+\bar{\nu}_{e}$, can be written as

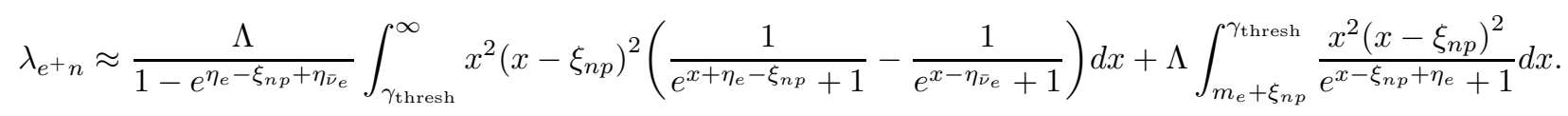

Again we see that if $\bar{\epsilon}<\xi_{n p}+m_{e}$, then from Eq. A12 the threshold is $\gamma_{\text {thresh }}=\xi_{n p}+m_{e}$ and the neutrino flavor conversion will have no affect on the rate. In this case, the second term of Eq. A13 will vanish and the first term will be the rate with no neutrino conversion. The full rate expression in Eq. A13 can be broken up into three parts,

$$
\lambda_{e^{+} n}=\lambda_{e^{+} n}^{\mathrm{first}}+\lambda_{e^{+} n}^{\mathrm{snd}}+\lambda_{e^{+} n}^{\mathrm{thrd}},
$$

each of which can be rendered in terms of standard relativistic Fermi integrals.

Here $\lambda_{e^{+} n}^{\text {first }}$ corresponds to the first integral in Eq. (A13). It can be reduced to

$$
\lambda_{e^{+} n}^{\mathrm{first}} \approx \frac{\Lambda}{1-e^{\eta_{e}-\xi_{n p}+\eta_{\bar{\nu}}}} \sum_{n=0}^{4} \bar{\alpha}_{n}\left[F_{n}\left(\eta_{\bar{e}}^{\mathrm{eff}}\right)-F_{n}\left(\eta_{\bar{\nu}}^{\mathrm{eff}}\right)\right]
$$

where the $\bar{\alpha}_{n}$ are as defined for Eq. A11, the effective positron degeneracy parameter is $\eta_{\bar{e}}^{\text {eff }} \equiv-\eta_{e}+\xi_{n p}-\bar{\epsilon}$, and the effective $\bar{\nu}_{e}$ degeneracy parameter in this case is $\eta_{\bar{\nu}}^{\mathrm{eff}} \equiv \eta_{\bar{\nu}_{e}}-\bar{\epsilon}$.
Note that the second integral in Eq. A13 is the sum $\lambda_{e^{+} n}^{\text {snd }}+\lambda_{e^{+} n}^{\text {thrd }}$. The last term in this sum can be approximated as

$$
\lambda_{e^{+} n}^{\mathrm{thrd}} \approx-\Lambda \sum_{n=0}^{4} \bar{\alpha}_{n} F_{n}\left(\xi_{n p}-\eta_{e}-\bar{\epsilon}\right)
$$

where the $\bar{\alpha}_{n}$ are the same as defined above for Eqs. A11 \& Eq. A15. In similar fashion we can express $\lambda_{e^{+} n}^{\text {snd }}$ in terms of standard relativistic Fermi integrals,

$$
\lambda_{e^{+} n}^{\mathrm{snd}} \approx \Lambda \sum_{n=0}^{4} \bar{\beta}_{n} F_{n}\left(-m_{e}-\eta_{e}\right)
$$

We define $x \equiv 2 m_{e}+\xi_{n p}$ and $y=m_{e}\left(m_{e}+\xi_{n p}\right)$, with $m_{e} \equiv m_{e} c^{2} / T$. With these definitions we can write the $\bar{\beta}_{n}$ in Eq. A17) as: $\bar{\beta}_{4} \equiv 1$, while $\bar{\beta}_{3} \equiv 2 x, \bar{\beta}_{2} \equiv x^{2}+2 y$, $\bar{\beta}_{1} \equiv 2 x y$, and $\bar{\beta}_{0} \equiv y^{2}$. 